\title{
Mono- and poly-therapy with benzodiazepines or Z-drugs: Results from a tertiary-care Addiction Unit study
}

\author{
Giovanni Mansueto $^{\mathrm{a}, \mathrm{b}, \mathrm{c}}$, Fabio Lugoboni ${ }^{\mathrm{d}}$, Rebecca Casari ${ }^{\mathrm{d}}$, Anna Bertoldi ${ }^{\mathrm{d}}$ and \\ Fiammetta $\operatorname{Cosci}^{\mathrm{a}, \mathrm{b}, \mathrm{e}, *}$ \\ ${ }^{\mathrm{a}}$ Department of Health Sciences, University of Florence, Florence, Italy \\ ${ }^{\mathrm{b}}$ Clinical Pharmacopsychology Laboratory, University of Florence, Florence, Italy \\ ${ }^{\mathrm{c}}$ Department of Psychology, Sigmund Freud University, Milan, Italy \\ ${ }^{\mathrm{d}}$ Addiction Medicine Unit, Department of Medicine, Verona University Hospital, Verona, Italy \\ ${ }^{\mathrm{e}}$ Department of Psychiatry \& Psychology, School for Mental Health \& Neuroscience, Maastricht \\ University, Maastricht, The Netherlands
}

Received 1 March 2021

Accepted 13 May 2021

\begin{abstract}
.
BACKGROUND: Using benzodiazepines (BZDs) or Z-drugs in poly-therapy is a critical issue.

OBJECTIVE: Identifying factors influencing the use of BZDs/Z-drugs in poly- vs mono-therapy in patients with or without substance use disorders (SUDs).

METHODS: 986 inpatients were analysed. Socio-demographic and clinical variables were collected. BZD/Z-drug doses were compared via the Defined Daily Dose (DDD) and standardized as diazepam dose equivalents. Mann-Whitney, Chi-square, Fisher test, hierarchical multivariate regression analyses were run referring to the whole sample and to subjects with current SUDs, lifetime SUDs, current and lifetime SUDs, non-SUDs.

RESULTS: In the whole sample the variance of being mono- vs poly-therapy users was explained by BZD/Z-drug formulation, DDD, duration of treatment, age of first BZDs/Z-drugs use $\left(\Delta R^{2}=0.141, p<0.001\right)$. Among those with current SUDs $\left(\Delta R^{2}\right.$ $=0.278, p=0.332)$ or current and lifetime SUDs $\left(\Delta R^{2}=0.154, p=0.419\right)$, no variables explained the variance of being mono-vs poly-therapy users. Among lifetime SUDs subjects, the variance of being mono- vs poly-therapy users was explained by BZD/Zdrug formulation and age of first BZD/Z-drug use $\left(\Delta R^{2}=0.275, p<0.001\right)$. Among non-SUDs subjects, the variance of being mono- vs poly-therapy users was explained by DDD and duration of treatment $\left(\Delta R^{2}=0.162, p=0.001\right)$.

CONCLUSIONS: Tablets, high drug doses, long duration of treatment, and early age of first use were more likely associated to poly- than mono-therapy. This suggests that patients have different clinical features and a pharmacological prescription should be tailored to them also based on the variables here analysed.
\end{abstract}

Keywords: Mono-therapy, poly-therapy, poly-pharmacy, substance use disorder, benzodiazepine, Z-drug

\footnotetext{
*Address for correspondence: Fiammetta Cosci, Department of Health Sciences, University of Florence, via di San Salvi 12, 50135, Florence, Italy. Tel.: +39 055623 7811; E-mail: fiammetta.cosci@unifi.it.
} 


\section{Introduction}

Benzodiazepines (here abbreviated as BZDs) and non-benzodiazepine receptor agonists (here abbreviated as Z-drugs) are currently prescribed with prevalence rates in the general population ranging from $2 \%$ to $17 \%$ for BZDs [1-3] and from $2 \%$ to $9 \%$ for Z-drugs [2,4]. BZDs and Z-drugs are used in monotherapy or in poly-therapy; prevalence rates in the general population range from $23 \%$ to $44 \%$ [5,6] for mono-therapy and from $9 \%$ to $29 \%[2,4,5,7]$ for poly-therapy.

Of course, mono- and poly-therapy users differ for clinical features. Poly-therapy users are more prevalent than mono-therapy users when the duration of the treatment is longer or the dose is higher $[4,6,8,9]$. Poly-therapy users have higher rates of adverse events, toxicity, harmful drug-to drug interactions, and increased risk of mortality, including the one due to suicide, than mono-therapy users [10-12]. Polytherapy users are highly represented among Substance Use Disorder (SUD) patients, with $34.6 \%$ using more than one BZD and 20\% using both BZDs and Z-drugs [9].

Unfortunately, the investigation of the potential factors influencing the risk of using BZDs or Z-drugs in poly-therapy rather than in mono-therapy has not received enough attention. A recent study conducted in the general population found that current depressive symptoms and obesity are associated with two-fold increased risk of BZD/Z-drug use in poly-therapy than in mono-therapy [13] while no studies have been run among SUDs patients.

In this framework, we examined mono-therapy and poly-therapy users with the aim of identifying sociodemographic and clinical characteristics influencing the risk of using BZDs or Z-drugs in poly-therapy, versus mono-therapy, in patients with or without SUDs.

\section{Methods}

\subsection{Participants}

BZD/Z-drug dependent patients consecutively admitted at the Addiction Unit (AU) of the Verona University Hospital (Verona, Italy) from January 2003 to June 2019 were screened. Subjects had to meet the following inclusion criteria: 1 . age $\geq 18$ years; 2 . a diagnosis of BZD/Z-drug dependence according to the DSM-IV-TR criteria [14] lasting from at least 180 days [3] (the DSM-IV-TR diagnosis of dependence is based on at least 3 dependence criteria while the DSM-5 diagnosis of substance use disorder is based on at least 2 substance use disorder criteria which may include both abuse and dependence criteria according to the DSM-IV-TR); 3. a BZD/Z-drug daily consumption $>5$ times the maximum daily dose recommended (i.e., $50 \mathrm{mg}$ daily diazepam dose equivalent) $[15,16]$.

Written informed consent was obtained from all eligible patients. The study protocol fully adhered to the guidelines of the Ethic Committee of the Verona University Hospital.

\subsection{Procedure}

Socio-demographic (i.e., age, sex, marital status, education, working activity) and clinical data (i.e., smoking status, BZD/Z-drug used, BZD/Z-drug average daily dose in the last 180 days, duration of $\mathrm{BZD} / Z$-drug use, age of $\mathrm{BZD} / \mathrm{Z}$-drug first use, formulation of $\mathrm{BZD} / \mathrm{Z}$-drug used) were collected via a set of interview-based screening questions already used in the past [17]. Psychiatric disorders were assessed via the Structure Clinical Interview for DSM-IV - Patient Version (SCID-I-P) [18]. The SCID-I$P$ showed excellent validity and reliability [18]. The Italian version is consistent with the English one [19]. 
Personality disorders were assessed via the semi-structured Diagnostic Interview for DSM-IV Personality Disorders (DIPD-IV) [20]. The DIDP-IV showed good inter-rater and test-retest reliability [20].

\subsection{Statistical analyses}

The Kolmogorov-Smirnov test was run to test normality of data [21]. Given that data were nonnormally distributed, Mann-Whitney matched pairs test was used to compare rank means of continuous variables [22]. Chi-square tests or Fisher test, when more than $20 \%$ of cells had expected frequencies less than 5 [23], were run to compare rates.

BZD/Z-drug doses were compared via the Defined Daily Dose (DDD) (i.e., the therapeutic daily dose according to the World Health Organization) [24], BZD/Z-drug dose was standardized as diazepam dose equivalents according to conversion tables $[25,26]$.

Subjects were stratified based on the number of BDZs/Z-drugs used. Mono-therapy users were defined as those who used only one BZD or Z-drug, poly-therapy users were defined as those who used more than one BZD or Z-drug [27]. The comparisons between mono- and poly-therapy users were run for the whole sample as well as separately for groups of patients with current SUDs, lifetime SUDs, current and lifetime SUDs, or without SUDs. Since tobacco smoking appears to be different to other substances in terms of tolerance, time spent to have it or to dispose of the effect, hazardous use, perceived difficulty quitting, and toxic effects [28,29], cigarette smoking was not considered as a SUD.

Hierarchical multivariate regression analyses were run to identify predicting variables influencing the risk of using BZDs or Z-drugs in mono-therapy or in poly-therapy for the whole sample as well as separately for the groups described above. Mono-therapy vs poly-therapy users was used as reference. The entry order of predicting variables was the following for all regressions (this methodological choice was taken to increase the comparability of the results): sex, working activity, education, lifetime alcohol use, current and lifetime barbiturate use were entered at Step 1 as adjusting variables; duration of BZD/Zdrug use and diazepam equivalent were entered respectively at Steps 2 and 3 since they significantly differed between mono- and poly-therapy users in one out of the five comparisons taken into account (i.e., the whole sample, patients with current SUDs, patients with lifetime SUDs, patients with current and lifetime SUDs, patients without SUDs); daily dose, which significantly differed between mono- and poly-therapy users in 2 out of 5 comparisons, was entered at Step 4; DDD, which significantly differed between mono- and poly-therapy users in 3 out of 5 comparisons, was entered at Step 5; age of first BZD/Zdrug use, which significantly differed between mono- and poly-therapy users in 4 out of 5 comparisons, was entered at Step 6; formulation of BZD/Z-drug used (i.e., tablets or drops), which significantly differed between mono- and poly-therapy users in all comparisons, was entered at Step 7. Two-sided signicance level was set at $p \leq 0.05$. Analyses were performed via SPSS, version 21 (SPSS Inc., Chicago, IL, USA).

\section{Results}

A total of $1,112 \mathrm{BZD} / \mathrm{Z}$-drug users were consecutively enrolled at the AU of the Verona University Hospital (Verona, Italy) from January 2003 to June 2019. Among them, 126 were excluded because using both tablets and drops $(n=109)$ or had missing data on the formulation information $(n=17)$.

A total of 986 subjects were analysed, $515(52.23 \%)$ were males and $471(47.77 \%)$ females, the mean age was $44.95 \pm 10.83$ years. Table 1 shows demographic and clinical characteristics of the whole sample. Most of the subjects were unmarried, had a high school degree, and were employed. A total of $112(11.36 \%)$ subjects had a current SUD, $231(23.43 \%)$ a lifetime SUD, $183(18.56 \%)$ both current 
Table 1

Demographic and clinical variables. Total sample $(n=986)$

\begin{tabular}{|c|c|}
\hline & $n(\%)$ \\
\hline \multicolumn{2}{|l|}{ DEMOGRAPHIC VARIABLES } \\
\hline \multicolumn{2}{|l|}{ Marital status } \\
\hline Married & $284(28.80 \%)$ \\
\hline Unmarried & $508(51.52 \%)$ \\
\hline Cohabitant & $90(9.13 \%)$ \\
\hline Widower & $14(1.42 \%)$ \\
\hline Missing & $90(9.13 \%)$ \\
\hline \multicolumn{2}{|l|}{ Education } \\
\hline Primary school & $55(5.58 \%)$ \\
\hline Secondary school & $290(29.41 \%)$ \\
\hline High school & $392(39.76 \%)$ \\
\hline Graduation & $192(19.47 \%)$ \\
\hline Missing & $57(5.78 \%)$ \\
\hline \multicolumn{2}{|l|}{ Working status } \\
\hline Employed & $488(49.49 \%)$ \\
\hline Unemployed & $275(27.89 \%)$ \\
\hline Housewife/retired/invalid & $198(20.08 \%)$ \\
\hline Missing & $25(2.54 \%)$ \\
\hline \multicolumn{2}{|l|}{ CLINICAL VARIABLES } \\
\hline \multicolumn{2}{|l|}{ Substance use disorder } \\
\hline Current substance use disorder & $112(11.36 \%)$ \\
\hline Alcohol use & $75(7.61 \%)$ \\
\hline Cocaine use & $25(2.55 \%)$ \\
\hline THC use & $16(1.62 \%)$ \\
\hline Heroin use & $6(0.61 \%)$ \\
\hline Barbiturates use & $4(0.40 \%)$ \\
\hline Lifetime substance use disorder & $231(23.43 \%)$ \\
\hline Alcohol use & $138(13.99 \%)$ \\
\hline Cocaine use & $113(11.46 \%)$ \\
\hline THC use & $89(9.03 \%)$ \\
\hline Heroin use & $74(7.51 \%)$ \\
\hline Barbiturates use & $0(0.00 \%)$ \\
\hline Current and lifetime substance use disorder & $183(18.56 \%)$ \\
\hline Alcohol use & $124(12.58 \%)$ \\
\hline Cocaine use & $142(14.40 \%)$ \\
\hline THC use & $119(12.07 \%)$ \\
\hline Heroin use & $107(10.85 \%)$ \\
\hline Barbiturates use & $5(0.51 \%)$ \\
\hline No substance use disorder & $460(46.65 \%)$ \\
\hline \multicolumn{2}{|l|}{ Smoking status } \\
\hline Current smokers & $637(64.60 \%)$ \\
\hline Past smokers & $75(7.61 \%)$ \\
\hline Non smokers & $255(25.86 \%)$ \\
\hline Missing & $19(1.93 \%)$ \\
\hline
\end{tabular}


Table 1 (Continued).

\begin{tabular}{|c|c|}
\hline & $n(\%)$ \\
\hline \multicolumn{2}{|l|}{ Current psychiatric disorder } \\
\hline Present & $901(91.38 \%)$ \\
\hline Absent & $84(8.52 \%)$ \\
\hline Missing & $1(0.10 \%)$ \\
\hline \multicolumn{2}{|c|}{ Type of current psychiatric disorder } \\
\hline Anxiety/depressive disorders & $767(77.79 \%)$ \\
\hline Personality disorders & $116(11.76 \%)$ \\
\hline Eating disorders & $6(0.61 \%)$ \\
\hline Psychotic disorder & $5(0.51 \%)$ \\
\hline ADHD & $4(0.40 \%)$ \\
\hline Obsessive compulsive disorder & $3(0.30 \%)$ \\
\hline Other & $19(1.93 \%)$ \\
\hline Missing & $94(9.53 \%)$ \\
\hline \multicolumn{2}{|l|}{ BZD used } \\
\hline Lormetazepam & $580(58.82 \%)$ \\
\hline Lorazepam & $142(14.40 \%)$ \\
\hline Alprazolam & $118(11.97 \%)$ \\
\hline Clonazepam & $42(4.26 \%)$ \\
\hline Bromazepam & $39(3.95 \%)$ \\
\hline Triazolam & $39(3.95 \%)$ \\
\hline Diazepam & $34(3.45 \%)$ \\
\hline Delorazepam & $21(2.13 \%)$ \\
\hline Flurazepam & $12(1.22 \%)$ \\
\hline Etizolam & $9(0.91 \%)$ \\
\hline Flunitrazepam & $4(0.40 \%)$ \\
\hline Oxazepam & $3(0.30 \%)$ \\
\hline Temazepam & $2(0.20 \%)$ \\
\hline Brotizolam & $2(0.20 \%)$ \\
\hline Prazepam & $2(0.20 \%)$ \\
\hline Ketazolam & - \\
\hline \multicolumn{2}{|l|}{ Z-drugs used } \\
\hline Zolpidem & $107(10.85 \%)$ \\
\hline Zoplicone & $3(0.30 \%)$ \\
\hline \multicolumn{2}{|l|}{ Formulation of BZD/Z-drugs used } \\
\hline Tablets & $298(30.22 \%)$ \\
\hline Drops & $688(69.78 \%)$ \\
\hline
\end{tabular}

Note ADHD: attention deficit hyperactivity disorder; BZD: benzodiazepine; Z-drugs: non-benzodiazepine receptor agonists.

and lifetime SUDs, 460 (46.65\%) did not report SUDs. Cocaine and alcohol had the highest rates of current/lifetime use. The majority were current smokers $(n=637,64.60 \%)$ and had at least one current psychiatric disorder $(n=901,91.38 \%)$.

Among BZDs, lormetazepam had the highest rate of use, followed by lorazepam, and alprazolam. Among Z-drugs, zolpidem had the highest rate of use. 
Table 2

Comparison between mono-therapy users $(n=846)$ and poly-therapy users $(n=140)$. Chi-square and Fisher test

\begin{tabular}{|c|c|c|c|c|c|c|}
\hline & & Mono-therapy users & & Poly-therapy users & & \\
\hline & $n$ & $n(\%)$ & $n$ & & Chi-square (df) & $p$ \\
\hline DEMOGRAPHIC VARIABL & & & & & & \\
\hline Marital status & 774 & & 122 & & & \\
\hline Married & & 247 (31.91\%) & & $37(30.33 \%)$ & $0.15_{(3)}$ & 0.985 \\
\hline Unmarried & & $437(56.46 \%)$ & & $71(58.20 \%)$ & & \\
\hline Cohabitant & & $78(10.08 \%)$ & & $12(9.84 \%)$ & & \\
\hline Widower & & $12(1.55 \%)$ & & $2(1.63 \%)$ & & \\
\hline Education & 801 & & 128 & & & \\
\hline Primary school & & $48(5.99 \%)$ & & $7(5.47 \%)$ & $1.15_{(3)}$ & 0.764 \\
\hline Secondary school & & $252(31.46 \%)$ & & $38(29.69 \%)$ & & \\
\hline High school & & $340(42.45 \%)$ & & $52(40.62 \%)$ & & \\
\hline Graduation & & $161(20.10 \%)$ & & $31(24.22 \%)$ & & \\
\hline Working status & 824 & & & & & \\
\hline Employed & & $431(52.30 \%)$ & & $57(41.60 \%)$ & $6.26_{(2)}$ & 0.044 \\
\hline Unemployed & & $232(28.16 \%)$ & & $43(31.39 \%)$ & & \\
\hline Housewife or retired or invalid & & $161(19.54 \%)$ & & $37(27.01 \%)$ & & \\
\hline CLINICAL VARIABLES & & & & & & \\
\hline Substance use disorder & 846 & & 140 & & & \\
\hline Current & & $95(11.23 \%)$ & & $17(12.14 \%)$ & $3.18_{(3)}$ & 0.364 \\
\hline Lifetime & & $192(22.70 \%)$ & & $39(27.86 \%)$ & & \\
\hline Lifetime and current & & $155(18.32 \%)$ & & $28(20.00 \%)$ & & \\
\hline Absent & & $404(47.75 \%)$ & & $56(40.00 \%)$ & & \\
\hline Smoking status & 830 & & 137 & & & \\
\hline Current smokers & & $547(65.90 \%)$ & & $90(65.69 \%)$ & $4.02_{(2)}$ & 0.134 \\
\hline Past smokers & & $59(7.11 \%)$ & & $16(11.68 \%)$ & & \\
\hline Non smokers & & $224(26.99 \%)$ & & $31(22.63 \%)$ & & \\
\hline Current psychiatry disorder & 845 & & 140 & & & \\
\hline Present & & $769(91.01 \%)$ & & $132(94.29 \%)$ & $1.66_{(1)}$ & 0.198 \\
\hline Absent & & $76(8.99 \%)$ & & $8(5.71 \%)$ & & \\
\hline BZD used & & & & & & \\
\hline Lormetazepam & & & 140 & & & \\
\hline Yes & & $519(61.35 \%)$ & & $61(43.57 \%)$ & $15.67_{(1)}$ & $<0.001$ \\
\hline No & & $327(38.65 \%)$ & & $79(56.43 \%)$ & & \\
\hline Lorazepam & 846 & & 140 & & & \\
\hline & & $90(10.64 \%)$ & & $52(37.14 \%)$ & $68.45_{(1)}$ & $<0.001$ \\
\hline No & & $756(89.36 \%)$ & & $88(62.86 \%)$ & & \\
\hline Alprazolam & 846 & & 140 & & & \\
\hline & & $76(8.98 \%)$ & & $42(30.00 \%)$ & $50.36_{(1)}$ & $<0.001$ \\
\hline No & & 770 (91.02\%) & & $98(70.00 \%)$ & & \\
\hline
\end{tabular}


Table 2 (Continued).

\begin{tabular}{|c|c|c|c|c|c|c|}
\hline & $n$ & $\begin{array}{l}\text { Mono-therapy users } \\
n(\%)\end{array}$ & $n$ & $\begin{array}{l}\text { Poly-therapy users } \\
n(\%)\end{array}$ & Chi-square $_{(\mathrm{df})}$ & $p$ \\
\hline Clonazepam & 846 & & 140 & & & \\
\hline Yes & & $17(2.01 \%)$ & & $25(17.86 \%)$ & $73.97(1)$ & $<0.001$ \\
\hline No & & $829(97.99 \%)$ & & $115(82.14 \%)$ & & \\
\hline Bromazepam & 846 & & 140 & & & \\
\hline Yes & & $24(2.84 \%)$ & & $15(10.71 \%)$ & $19.62_{(1)}$ & $<0.001$ \\
\hline No & & $822(97.16 \%)$ & & $125(89.28 \%)$ & & \\
\hline Triazolam & 846 & & 140 & & & \\
\hline Yes & & $16(1.89 \%)$ & & $23(16.43 \%)$ & $66.82_{(1)}$ & $<0.001$ \\
\hline No & & $830(98.11 \%)$ & & 117 (83.57\%) & & \\
\hline Diazepam & 846 & & 140 & & & \\
\hline Yes & & $13(1.54 \%)$ & & $21(15.00 \%)$ & $65.40_{(1)}$ & $<0.001$ \\
\hline No & & $833(98.46 \%)$ & & $119(85.00 \%)$ & & \\
\hline Delorazepam & 846 & & 140 & & & \\
\hline Yes & & $8(0.95 \%)$ & & $13(9.29 \%)$ & $40.08_{(1)}$ & $<0.001$ \\
\hline No & & $838(99.05 \%)$ & & $127(90.71 \%)$ & & \\
\hline Flurazepam & 846 & & 140 & & & \\
\hline Yes & & $0(0.00 \%)$ & & $12(8.57 \%)$ & $73.41_{(1)}$ & $<0.001^{\mathrm{a}}$ \\
\hline No & & $846(100.00 \%)$ & & $128(91.43 \%)$ & & \\
\hline Etizolam & 846 & & 140 & & & \\
\hline Yes & & $7(0.83 \%)$ & & $2(1.43 \%)$ & $0.48_{(1)}$ & $0.516^{\mathrm{a}}$ \\
\hline No & & $839(99.17 \%)$ & & $138(98.57 \%)$ & & \\
\hline Flunitrazepam & 846 & & 140 & & & \\
\hline Yes & & $0(0.00 \%)$ & & $4(2.86 \%)$ & $24.27_{(1)}$ & $<0.001^{\mathrm{a}}$ \\
\hline No & & $846(100.00 \%)$ & & $136(97.14 \%)$ & & \\
\hline Oxazepam & 846 & & 140 & & & \\
\hline Yes & & $0(0.00 \%)$ & & $3(2.14 \%)$ & $19.18_{(1)}$ & $\mathbf{0 . 0 0 3}^{\mathrm{a}}$ \\
\hline No & & $846(100.00 \%)$ & & 137 (97.86\%) & & \\
\hline Temazepam & 846 & & 140 & & & \\
\hline Yes & & $12 \%)$ & & $1(0.71 \%)$ & $2.11_{(1)}$ & $0.264^{\mathrm{a}}$ \\
\hline No & & 845 (99.88\%) & & $139(99.29 \%)$ & & \\
\hline Brotizolam & 846 & & 140 & & & \\
\hline Yes & & $1(0.12 \%)$ & & $1(0.71 \%)$ & $2.11_{(1)}$ & $0.264^{\mathrm{a}}$ \\
\hline No & & 845 (99.88\%) & & $139(99.29 \%)$ & & \\
\hline Prazepam & 846 & & 140 & & & \\
\hline Yes & & $1(0.12 \%)$ & & $1(0.71 \%)$ & $2.11_{(1)}$ & $0.264^{\mathrm{a}}$ \\
\hline No & & 845 (99.88\%) & & $139(99.29 \%)$ & & \\
\hline \multicolumn{7}{|l|}{ Z-drugs used } \\
\hline Zolpidem & 846 & & 140 & & & \\
\hline Yes & & $70(8.27 \%)$ & & $37(26.43 \%)$ & $40.92_{(1)}$ & $<0.001$ \\
\hline No & & $776(91.73 \%)$ & & $103(73.57 \%)$ & & \\
\hline
\end{tabular}


Table 2 (Continued).

\begin{tabular}{|c|c|c|c|c|c|c|}
\hline & $n$ & $\begin{array}{c}\text { Mono-therapy users } \\
n(\%)\end{array}$ & $n$ & $\begin{array}{c}\text { Poly-therapy users } \\
n(\%)\end{array}$ & Chi-square $_{(\mathrm{df})}$ & $p$ \\
\hline Zoplicone & 846 & & 140 & & & \\
\hline Yes & & $2(0.24 \%)$ & & $1(0.71 \%)$ & $0.90_{(1)}$ & 0.342 \\
\hline No & & $844(99.76 \%)$ & & $139(99.29 \%)$ & & \\
\hline Formulation of BZD/Z-drugs used & 846 & & 140 & & & \\
\hline Tablets & & $221(26.12 \%)$ & & $77(55.00 \%)$ & $47.50_{(1)}$ & $<0.001$ \\
\hline Drops & & $625(73.88 \%)$ & & $63(45.00 \%)$ & & \\
\hline
\end{tabular}

Note: BZD: benzodiazepine; Z-drugs: non-benzodiazepine receptor agonists; $a$ = Fisher test.

BZDs/Z-drugs were more frequently consumed in drops than in tablets, with a mean daily dose of $100.39 \pm 197.19 \mathrm{mg}$, a mean DDD of $52.20 \pm 64.15$, and a mean diazepam equivalent dose of $357.31 \pm$ $483.80 \mathrm{mg}$. The use of BZDs/Z-drugs lasted $86.30 \pm 86.07$ months. The mean age of first use was 30.44 \pm 10.52 years. In the whole sample, $846(85.80 \%)$ subjects were mono-therapy users while $140(14.20 \%)$ were poly-therapy users.

When mono-therapy users were compared with poly-therapy users, no statistically significant differences were found for sex (males: $448,52.96 \%$ vs $67,47.86 \%, x_{(\mathrm{df})}^{2}=1.25_{(1)}, p=0.263$ ) and age (44.96 \pm 10.85 vs $44.85 \pm 10.77$ years, $p=0.944)$. Table 2 shows the comparisons on demographic and clinical variables. Being employed was more represented among mono- than poly-therapy users. Lormetazepam was more frequently used by mono-therapy users; vice versa zolpidem. BZD/Z-drug drops were more likely used by mono- than poly-therapy users. Mono-therapy users showed lower daily dose $(99.62$ \pm 194.89 vs $105.02 \pm 211.31 \mathrm{mg}, p=0.005)$, higher DDD (54.33 \pm 65.64 vs $38.73 \pm 52.03, p<0.001)$, older age of first use (mean \pm SD: $30.97 \pm 10.45$ vs $27.19 \pm 10.45$ years, $p<0.001$ ) than poly-therapy users. The two groups did not differ for diazepam equivalent dose (354.67 \pm 500.46 vs $375.26 \pm 350.82$, $p=0.350)$ or duration of use $(82.67 \pm 81.15$ vs $109.57 \pm 110.16$ months, $p=0.058)$.

Among subjects with current SUDs $(\hat{n}=112), 95(84.82 \%)$ were mono- and $17(15.18 \%)$ poly-therapy users. No statistically significant differences were found for sex (males: $47,49.47 \%$ vs $7,41.18 \%$, $\left.x_{(\mathrm{df})}^{2}=0.39_{(1)}, p=0.528\right)$ and age (mean \pm SD: $45.67 \pm 11.09$ vs $45.94 \pm 10.36$ years, $p=0.761$ ). Table 3 shows the comparisons on demographic and clinical variables. Concerning BZDs use, lormetazepam was more likely used by mono-therapy users than poly-therapy users while, among Z-drugs, zolpidem was less frequently used by mono- than poly-therapy users. BZD/Z-drug drops were more likely used by monotherapy users. Mono-therapy users showed statistically significant lower diazepam equivalent dose (mean \pm SD: $295.55 \pm 357.77$ vs $383.84 \pm 251.11, p=0.014$ ) and older age of first use (mean \pm SD: 31.88 \pm 11.28 vs $23.62 \pm 11.03$ years, $p=0.019)$ than poly-therapy users. No differences were found for daily dose (mean \pm SD: $56.98 \pm 72.88$ vs $121.09 \pm 222.92 \mathrm{mg}, p=0.852$ ), DDD (mean \pm SD: $47.52 \pm 64.86$ vs $38.43 \pm 49.66, p=0.476$ ), duration of use (mean \pm SD: $85.56 \pm 76.12$ vs $73.79 \pm 101.52$ months, $p=0.147)$.

Among subjects with lifetime SUDs $(n=231), 192(83.12 \%)$ were mono-therapy users and 39 polytherapy users $(16.88 \%)$. No statistically significant differences were found for sex (males: 124, 64.58\% vs $21,53.85 \%, x_{(\mathrm{df})}^{2}=1.60_{(1)}, p=0.206$ ) and age (mean $\pm \mathrm{SD}: 45.14 \pm 10.76$ vs $45.69 \pm 10.72$ years, $p=0.674$ ). Table 4 shows the comparisons concerning demographic and clinical variables. The lifetime rate of alcohol use was significantly lower among mono- than poly-therapy users. Lorazepam, alprazolam, 
Table 3

Comparison between mono-therapy users $(n=95)$ and poly-therapy users $(n=17)$ among subjects with current substance use disorder. Chi-square and Fisher test

\begin{tabular}{|c|c|c|c|c|c|c|}
\hline & $n$ & $\begin{array}{c}\text { Mono-therapy users } \\
n(\%)\end{array}$ & $n$ & $\begin{array}{c}\text { Poly-therapy users } \\
n(\%)\end{array}$ & Chi-square $_{(\mathrm{df})}$ & $p$ \\
\hline \multicolumn{7}{|l|}{ DEMOGRAPHIC VARIABLES } \\
\hline Marital status & 83 & & 14 & & \multirow{5}{*}{$2.25_{(1)}$} & \multirow{5}{*}{0.521} \\
\hline Married & & $25(30.12 \%)$ & & $7(50.00 \%)$ & & \\
\hline Unmarried & & $50(60.24 \%)$ & & $6(42.86 \%)$ & & \\
\hline Cohabitant & & $7(8.43 \%)$ & & $1(7.14 \%)$ & & \\
\hline Widower & & $1(1.20 \%)$ & & $0(0.00 \%)$ & & \\
\hline Education & 87 & & 16 & & \multirow{5}{*}{$8.97_{(3)}$} & \multirow{5}{*}{0.030} \\
\hline Primary school & & $0(0.00 \%)$ & & $1(6.25 \%)$ & & \\
\hline Secondary school & & $22(25.29 \%)$ & & $3(18.75 \%)$ & & \\
\hline High school & & $40(45.97 \%)$ & & $4(25.00 \%)$ & & \\
\hline Graduation & & $25(28.74 \%)$ & & $8(50.00 \%)$ & & \\
\hline Working status & 91 & & & & \multirow{4}{*}{$0.950_{(2)}$} & \multirow{4}{*}{0.622} \\
\hline Employed & & $51(56.04 \%)$ & & $9(52.94 \%)$ & & \\
\hline Unemployed & & $23(25.27 \%)$ & & $6(35.29 \%)$ & & \\
\hline Housewife or retired or invalid & & $17(18.68 \%)$ & 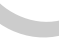 & $2(11.76 \%)$ & & \\
\hline \multicolumn{7}{|l|}{ CLINICAL VARIABLES } \\
\hline $\begin{array}{l}\text { Substance used currently } \\
\text { Heroin }\end{array}$ & 93 & & 17 & & & \\
\hline Yes & & $4(4.30 \%)$ & & $2(11.76 \%)$ & \multirow{2}{*}{$1.55_{(1)}$} & \multirow[t]{2}{*}{$0.232^{\mathrm{a}}$} \\
\hline No & & $89(95.70 \%)$ & & $15(88.23 \%)$ & & \\
\hline Cocaine & 93 & & 17 & & \multirow{3}{*}{$3.79_{(1)}$} & \multirow{3}{*}{$0.064^{\mathrm{a}}$} \\
\hline Yes & & $18(19.57 \%)$ & & $7(41.18 \%)$ & & \\
\hline No & & $74(80.43 \%)$ & & $10(58.82 \%)$ & & \\
\hline THC & 93 & & 17 & & \multirow{3}{*}{$1.31_{(1)}$} & \multirow{3}{*}{$0.268^{\mathrm{a}}$} \\
\hline Yes & & $12(12.90 \%)$ & & $4(23.53 \%)$ & & \\
\hline No & & $81(87.10 \%)$ & & $13(76.47 \%)$ & & \\
\hline Alcohol & 93 & & 17 & & \multirow{3}{*}{$1.78_{(1)}$} & \multirow{3}{*}{0.182} \\
\hline & & $66(69.47 \%)$ & & $9(52.94 \%)$ & & \\
\hline No & & $29(30.53 \%)$ & & $8(47.06 \%)$ & & \\
\hline Barbiturates & 93 & & 17 & & \multirow{3}{*}{$0.74_{(1)}$} & \multirow{3}{*}{$1.000^{\mathrm{a}}$} \\
\hline Yes & & $4(4.21 \%)$ & & $0(0.00 \%)$ & & \\
\hline No & & $91(95.79 \%)$ & & $17(100.00 \%)$ & & \\
\hline Smoking status & 93 & & 17 & & \multirow{4}{*}{$0.85_{(2)}$} & \multirow{4}{*}{0.652} \\
\hline Current smokers & & $66(70.97 \%)$ & & $12(70.59 \%)$ & & \\
\hline Past smokers & & $4(4.30 \%)$ & & $0(0.00 \%)$ & & \\
\hline Non smokers & & $23(24.73 \%)$ & & $5(29.41 \%)$ & & \\
\hline Current psychiatry disorder & 94 & & 17 & & \multirow{3}{*}{$1.01_{(1)}$} & \multirow{3}{*}{$0.291^{\mathrm{a}}$} \\
\hline Present & & $89(94.68 \%)$ & & $15(88.24 \%)$ & & \\
\hline Absent & & $5(5.32 \%)$ & & $2(11.76 \%)$ & & \\
\hline
\end{tabular}


Table 3 (Continued).

\begin{tabular}{|c|c|c|c|c|c|c|}
\hline & $n$ & $\begin{array}{l}\text { Mono-therapy users } \\
n(\%)\end{array}$ & $n$ & $\begin{array}{l}\text { Poly-therapy users } \\
n(\%)\end{array}$ & Chi-square $_{(\mathrm{df})}$ & $p$ \\
\hline \multicolumn{7}{|l|}{ BZD used } \\
\hline Lormetazepam & 95 & & 17 & & & \\
\hline Yes & & $61(64.21 \%)$ & & $5(29.41 \%)$ & $7.21_{(1)}$ & $0.014^{\mathrm{a}}$ \\
\hline No & & $34(35.79 \%)$ & & $12(70.59 \%)$ & & \\
\hline Lorazepam & 95 & & 17 & & & \\
\hline Yes & & $9(9.47 \%)$ & & $6(35.29 \%)$ & $8.29_{(1)}$ & $0.011^{\mathrm{a}}$ \\
\hline No & & $86(90.53 \%)$ & & $11(64.71 \%)$ & & \\
\hline Alprazolam & 95 & & 17 & & & \\
\hline Yes & & $12(12.63 \%)$ & & $5(29.41 \%)$ & $3.15_{(1)}$ & $0.133^{\mathrm{a}}$ \\
\hline No & & $83(87.37 \%)$ & & $12(70.59 \%)$ & & \\
\hline Clonazepam & 95 & & & 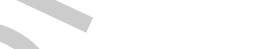 & & \\
\hline Yes & & $1(1.05 \%)$ & & $4(23.53 \%)$ & $17.08_{(1)}$ & $0.002^{\mathrm{a}}$ \\
\hline No & & $94(98.95 \%)$ & & $13(76.47 \%)$ & & \\
\hline Bromazepam & 95 & & 17 & & & \\
\hline Yes & & $1(1.05 \%)$ & & $1(5.88 \%)$ & $1.918_{(1)}$ & $0.282^{\mathrm{a}}$ \\
\hline No & & $94(98.95 \%)$ & & $16(94.12 \%)$ & & \\
\hline Triazolam & 95 & & 17 & & & \\
\hline Yes & & $1(1.05 \%)$ & & $4(23.53 \%)$ & $17.08_{(1)}$ & $0.002^{\mathrm{a}}$ \\
\hline No & & $94(98.95 \%)$ & & $13(76.47 \%)$ & & \\
\hline Diazepam & 95 & & 17 & & & \\
\hline Yes & & $2(2.11 \%)$ & & $2(11.76 \%)$ & $3.91_{(1)}$ & $0.109^{\mathrm{a}}$ \\
\hline No & & $93(97.89 \%)$ & & $15(88.24 \%)$ & & \\
\hline Delorazepam & 95 & & 17 & & & \\
\hline Yes & & $2(2.11 \%)$ & & $2(11.76 \%)$ & $3.91_{(1)}$ & $0.109^{\mathrm{a}}$ \\
\hline No & & $93(97.89 \%)$ & & $15(88.24 \%)$ & & \\
\hline Flurazepam & 95 & & 17 & & & \\
\hline Yes & & $0(0.00 \%)$ & & $0(0.00 \%)$ & - & - \\
\hline No & & $95(100.00 \%)$ & & $17(100.00 \%)$ & & \\
\hline Etizolam & 95 & & 17 & & & \\
\hline Yes & & $0(0.00 \%)$ & & $2(11.76 \%)$ & $11.38_{(1)}$ & $0.022^{\mathrm{a}}$ \\
\hline No & & $95(100.00 \%)$ & & $15(88.24 \%)$ & & \\
\hline Flunitrazepam & 9 & & 17 & & & \\
\hline Yes & & $0(0.00 \%)$ & & $1(5.88 \%)$ & $5.64_{(1)}$ & $0.152^{\mathrm{a}}$ \\
\hline No & & $95(100.00 \%)$ & & $16(94.12 \%)$ & & \\
\hline Oxazepam & 95 & & 17 & & & \\
\hline Yes & & $0(0.00 \%)$ & & $1(5.88 \%)$ & $5.64_{(1)}$ & $0.152^{\mathrm{a}}$ \\
\hline No & & $95(100.00 \%)$ & & $16(94.12 \%)$ & & \\
\hline Temazepam & 95 & & 17 & & & \\
\hline Yes & & $0(0.00 \%)$ & & $0(0.00 \%)$ & - & - \\
\hline No & & $95(100.00 \%)$ & & $17(100.00 \%)$ & & \\
\hline
\end{tabular}


Table 3 (Continued).

\begin{tabular}{|c|c|c|c|c|c|c|}
\hline & $n$ & $\begin{array}{l}\text { Mono-therapy users } \\
n(\%)\end{array}$ & $n$ & $\begin{array}{c}\text { Poly-therapy users } \\
n(\%)\end{array}$ & Chi-square $_{(\mathrm{df})}$ & $p$ \\
\hline Brotizolam & 95 & & 17 & & & \\
\hline Yes & & $0(0.00 \%)$ & & $0(0.00 \%)$ & - & - \\
\hline No & & $95(100.00 \%)$ & & $17(100.00 \%)$ & & \\
\hline Prazepam & 95 & & 17 & & & \\
\hline Yes & & $0(0.00 \%)$ & & $0(0.00 \%)$ & - & - \\
\hline No & & $95(100.00 \%)$ & & $17(100.00 \%)$ & & \\
\hline \multicolumn{7}{|l|}{ Z-drug used } \\
\hline Zolpidem & 95 & & 17 & & & \\
\hline Yes & & $6(6.32 \%)$ & & $7(41.18 \%)$ & $17.08_{(1)}$ & $0.001^{\mathrm{a}}$ \\
\hline No & & $89(93.68 \%)$ & & $10(58.82 \%)$ & & \\
\hline Zoplicone & 95 & & & & & \\
\hline Yes & & $95(100.00 \%)$ & & $17(100.00 \%)$ & - & - \\
\hline No & & $0(0.00 \%)$ & & $0(0.00 \%)$ & & \\
\hline Formulation of BZD/Z-drugs & 95 & & 17 & & & \\
\hline Tablets & & $25(26.32 \%)$ & & $10(58.82 \%)$ & $7.09_{(1)}$ & 0.008 \\
\hline Drops & & $70(73.68 \%)$ & & $7(41.18 \%)$ & & \\
\hline
\end{tabular}

Note: BZD: benzodiazepine; Z-drugs: non-benzodiazepine receptor agonists; a = Fisher test.

clonazepam, triazolam, diazepam, delorazepam, flurazepam, and oxazepam were used less frequently by mono- than poly-therapy users, vice versa zolpidem. BZD/Z-drug drops were more likely used by mono-therapy users. Mono-therapy users showed statistically significant higher DDD (mean \pm SD: 55.11 \pm 62.17 vs $40.83 \pm 54.38, p=0.045$ ) and older age of first use (mean \pm SD: $31.13 \pm 11.1$ vs $26.97 \pm 9.03$ years, $p=0.029$ ) than poly-therapy users. No differences were found for daily dose (mean \pm SD: 107.58 \pm 207.98 vs $122.76 \pm 183.01 \mathrm{mg}, p=0.805$ ), diazepam equivalent dose (mean \pm SD: $383.71 \pm 384.98$ vs $407.87 \pm 402.38, p=0.930$ ), duration of use (mean \pm SD: $85.07 \pm 82.57$ vs $100.62 \pm 100.86$ months, $p=0.739)$.

Among subjects with current and lifetime SUDs $(n=183), 155(84.70 \%)$ were mono-therapy users and $28(15.30 \%)$ poly-therapy users. Mono-therapy users were more frequently males (124 vs 17 , $\left.x_{(\mathrm{df})}^{2}=4.98_{(1)}, p=0.026\right)$ while age was not statistically significantly different $(40.39 \pm 8.92$ vs 40.68 \pm 8.60 years, $p=0.927$ ). Table 5 shows the comparisons concerning demographic and clinical variables. The rate of barbiturates use was lower among mono- than poly-therapy users. Concerning BZDs, lorazepam, alprazolam, clonazepam, triazolam, diazepam, delorazepam, and flurazepam were used less frequently by mono-therapy users, vice versa zolpidem.

BZD/Z-drug drops were used more likely by mono- than poly-therapy users. No statistically significant differences were observed between mono-therapy and poly-therapy users for daily dose (mean \pm SD: $102.58 \pm 211.87$ vs $68.42 \pm 90.25 \mathrm{mg}, p=0.100)$, DDD (mean \pm SD: $52.29 \pm 67.51$ vs $46.81 \pm 70.64, p$ $=0.123$ ), diazepam equivalent dose (mean \pm SD: $355.37 \pm 454.01$ vs $456.80 \pm 442.75, p=0.320)$, age of first use (mean \pm SD: $28.24 \pm 9.07$ vs $25.85 \pm 9.36$ years, $p=0.210$ ), duration of use (mean \pm SD: 62.56 \pm 65.76 vs $74.48 \pm 71.44$ months, $p=0.549$ ).

Among subjects without SUDs $(n=460), 404(87.83 \%)$ were mono-therapy users and $56(12.17 \%)$ poly-therapy users. No statistically significant differences were found for sex (males: $153,37.87 \%$ vs 
Table 4

Comparison between mono-therapy users $(n=192)$ and poly-therapy users $(n=39)$ among subjects with lifetime substance use disorder. Chi-square and fisher test

\begin{tabular}{|c|c|c|c|c|c|c|}
\hline & $n$ & $\begin{array}{l}\text { Mono-therapy users } \\
n(\%)\end{array}$ & $n$ & $\begin{array}{l}\text { Poly-therapy users } \\
n(\%)\end{array}$ & Chi-square $_{(\mathrm{df})}$ & $p$ \\
\hline \multicolumn{7}{|c|}{ DEMOGRAPHIC VARIABLES } \\
\hline Marital status & 184 & & 37 & & & \\
\hline Married & & $44(23.91 \%)$ & & $9(24.32 \%)$ & $0.959_{(3)}$ & 0.811 \\
\hline Unmarried & & $111(60.33 \%)$ & & $24(64.86 \%)$ & & \\
\hline Cohabitant & & $26(14.13 \%)$ & & $4(10.81 \%)$ & & \\
\hline Widower & & $3(1.63 \%)$ & & $0(0.00 \%)$ & & \\
\hline Education & 188 & & 37 & & & \\
\hline Primary school & & $16(8.51 \%)$ & & $0(0.00 \%)$ & $8.67_{(3)}$ & 0.034 \\
\hline Secondary school & & $70(37.23 \%)$ & & $8(21.62 \%)$ & & \\
\hline High school & & $73(38.83 \%)$ & & $22(59.46 \%)$ & & \\
\hline Graduation & & $29(15.42 \%)$ & & $7(18.92 \%)$ & & \\
\hline Working status & 192 & & & & & \\
\hline Employed & & $92(47.92 \%)$ & & $17(43.59 \%)$ & $4.54_{(2)}$ & 0.103 \\
\hline Unemployed & & $60(31.25 \%)$ & & $8(20.51 \%)$ & & \\
\hline Housewife or retired or invalid & & $40(20.83 \%)$ & & $14(35.90 \%)$ & & \\
\hline \multicolumn{7}{|l|}{ CLINICAL VARIABLES } \\
\hline Substance used lifetime & & & & & & \\
\hline Heroin & 192 & & 39 & & & \\
\hline Yes & & $63(32.81 \%)$ & & $11(28.21 \%)$ & & \\
\hline No & & $129(67.19 \%)$ & & $28(71.79 \%)$ & $0.316_{(1)}$ & 0.574 \\
\hline Cocaine & 192 & & 39 & & & \\
\hline Yes & & $97(50.52 \%)$ & & $16(41.03 \%)$ & $1.17_{(1)}$ & 0.279 \\
\hline No & & $95(49.48 \%)$ & & $23(58.97 \%)$ & & \\
\hline THC & 189 & & 39 & & & \\
\hline Yes & & $73(38.62 \% \%)$ & & $16(41.03 \%)$ & $0.078_{(1)}$ & 0.780 \\
\hline No & & $116(61.38 \% \%)$ & & $23(58.97 \% \%)$ & & \\
\hline Alcohol & 191 & & 39 & & & \\
\hline Yes & & $109(57.07 \%)$ & & $29(74.36 \%)$ & $4.03_{(1)}$ & 0.045 \\
\hline No & & $82(42.93 \%)$ & & $10(25.64 \%)$ & & \\
\hline Barbiturates & 191 & , & 39 & & & \\
\hline Yes & & $0(0.00 \%)$ & & $0(0.00 \%)$ & - & - \\
\hline No & & $191(100.00 \%)$ & & $39(100.00 \%)$ & & \\
\hline Smoking status & 192 & & 39 & & & \\
\hline Current smokers & & $162(84.38 \%)$ & & $28(71.79 \%)$ & $3.74_{(2)}$ & 0.154 \\
\hline Past smokers & & $17(8.85 \%)$ & & $7(17.95 \%)$ & & \\
\hline Non smokers & & $13(6.77 \%)$ & & $4(10.26 \%)$ & & \\
\hline Current psychiatry disorder & 192 & & 39 & & & \\
\hline Present & & $171(89.06 \%)$ & & $37(94.87 \%)$ & $1.22_{(1)}$ & $0.384^{\mathrm{a}}$ \\
\hline Absent & & $21(10.94 \%)$ & & $2(5.13 \%)$ & & \\
\hline
\end{tabular}


Table 4 (Continued).

\begin{tabular}{|c|c|c|c|c|c|c|}
\hline & $n$ & $\begin{array}{l}\text { Mono-therapy users } \\
n(\%)\end{array}$ & $n$ & $\begin{array}{l}\text { Poly-therapy users } \\
n(\%)\end{array}$ & Chi-square $_{(\mathrm{df})}$ & $p$ \\
\hline \multicolumn{7}{|l|}{ BZD used } \\
\hline Lormetazepam & 192 & & 39 & & & \\
\hline Yes & & $108(56.25 \%)$ & & $17(43.19 \%)$ & $2.09_{(1)}$ & 0.148 \\
\hline No & & $84(43.75 \%)$ & & $22(56.41 \%)$ & & \\
\hline Lorazepam & 192 & & 39 & & & \\
\hline Yes & & $22(11.46 \%)$ & & $15(38.46 \%)$ & $17.57(1)$ & $<0.001$ \\
\hline No & & $170(88.54 \%)$ & & $24(61.54 \%)$ & & \\
\hline \multicolumn{7}{|l|}{ Alprazolam } \\
\hline Yes & & $21(10.94 \%)$ & & $13(33.33 \%)$ & $12.95_{(1)}$ & $<0.001$ \\
\hline No & & $171(89.06 \%)$ & & $26(66.67 \%)$ & & \\
\hline Clonazepam & 192 & & & & & \\
\hline Yes & & $7(3.65 \%)$ & & $7(17.95 \%)$ & $11.64_{(1)}$ & $0.003^{\mathrm{a}}$ \\
\hline No & & $185(96.35 \%)$ & & $32(82.05 \%)$ & & \\
\hline Bromazepam & 192 & & 39 & & & \\
\hline Yes & & $3(1.56 \%)$ & & $3(7.69 \%)$ & $4.81_{(1)}$ & $0.062^{\mathrm{a}}$ \\
\hline No & & $189(98.44 \%)$ & & $36(92.31 \%)$ & & \\
\hline Triazolam & 192 & & 39 & & & \\
\hline Yes & & $7(3.65 \%)$ & 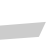 & $5(12.82 \%)$ & $5.54_{(1)}$ & $0.034^{\mathrm{a}}$ \\
\hline No & & $185(96.35 \%)$ & & $34(87.18 \%)$ & & \\
\hline Diazepam & 192 & & 39 & & & \\
\hline Yes & & $3(1.56 \%)$ & & $7(17.95 \%)$ & $21.02_{(1)}$ & $<0.001^{\mathrm{a}}$ \\
\hline No & & 189 (98.44\%) & & $32(82.05 \%)$ & & \\
\hline Delorazepam & 192 & & 39 & & & \\
\hline Yes & & $2(1.04 \%)$ & & $2(5.13 \%)$ & $3.18_{(1)}$ & $0.133^{\mathrm{a}}$ \\
\hline No & & $190(99.96 \%)$ & & 37 (94.87\%) & & \\
\hline Flurazepam & 192 & & 39 & & & \\
\hline Yes & & $0(0.00 \%)$ & & $4(10.26 \%)$ & $20.04_{(1)}$ & $0.001^{\mathrm{a}}$ \\
\hline No & & $192(100.00 \%)$ & & $35(89.74 \%)$ & & \\
\hline Etizolam & 192 & & 39 & & & \\
\hline Yes & & $3(1.56 \%)$ & & $0(0.00 \%)$ & $0.617_{(1)}$ & 0.432 \\
\hline No & & $189(98.44 \%)$ & & $39(100.00 \%)$ & & \\
\hline Flunitrazepam & 192 & & 39 & & & \\
\hline Yes & & $0(0.00 \%)$ & & $1(2.56 \%)$ & $4.94_{(1)}$ & $0.169^{\mathrm{a}}$ \\
\hline No & & $192(100.00 \%)$ & & 38 (97.44\%) & & \\
\hline Oxazepam & 192 & & 39 & & & \\
\hline Yes & & $0(0.00 \%)$ & & $1(2.56 \%)$ & $4.94_{(1)}$ & $0.169^{\mathrm{a}}$ \\
\hline No & & $192(100.00 \%)$ & & $38(97.46 \%)$ & & \\
\hline
\end{tabular}


Table 4 (Continued).

\begin{tabular}{|c|c|c|c|c|c|c|}
\hline & $n$ & $\begin{array}{l}\text { Mono-therapy users } \\
n(\%)\end{array}$ & $n$ & $\begin{array}{l}\text { Poly-therapy users } \\
n(\%)\end{array}$ & Chi-square $_{(\mathrm{df})}$ & $p$ \\
\hline Temazepam & 192 & & 39 & & & \\
\hline Yes & & $0(0.00 \%)$ & & $0(0.00 \%)$ & - & - \\
\hline No & & $192(100.00 \%)$ & & $39(100.00 \%)$ & & \\
\hline Brotizolam & 192 & & 39 & & & \\
\hline Yes & & $0(0.00 \%)$ & & $1(2.56 \%)$ & $4.94_{(1)}$ & $0.169^{\mathrm{a}}$ \\
\hline No & & $192(100.00 \%)$ & & $38(97.44 \%)$ & & \\
\hline Prazepam & 192 & & 39 & & & \\
\hline yes & & $0(0.00 \%)$ & & $1(2.56 \%)$ & $4.94_{(1)}$ & $0.169^{\mathrm{a}}$ \\
\hline No & & $192(100.00 \%)$ & & 38 (97.44\%) & & \\
\hline \multicolumn{7}{|l|}{ Z-drugs used } \\
\hline Zolpidem & 192 & & & & & \\
\hline Yes & & $16(8.33 \%)$ & & $11(28.20 \%)$ & $12.40_{(1)}$ & $0.001^{\mathrm{a}}$ \\
\hline No & & $176(91.67 \%)$ & & $28(71.79 \%)$ & & \\
\hline Zoplicone & 192 & & & & & \\
\hline Yes & & $0(0.00 \%)$ & & $0(0.00 \%)$ & - & - \\
\hline No & & $192(100.00 \%)$ & & $39(100.00 \%)$ & & \\
\hline $\begin{array}{l}\text { Formulation of } \mathrm{BZD} / \mathrm{Z} \text {-drugs } \\
\text { used }\end{array}$ & 192 & & 39 & & & \\
\hline Tablets & & $57(29.69 \%)$ & & $23(58.97 \%)$ & $12.28_{(1)}$ & $0.001^{\mathrm{a}}$ \\
\hline Drops & & $135(70.31 \%)$ & & $16(41.03 \%)$ & & \\
\hline
\end{tabular}

Note: BZD: benzodiazepine; Z-drugs: non-benzodiazepine receptor agonists; a = Fisher test.

$\left.22,30.28 \%, x_{(\mathrm{df})}^{2}=0.04_{(1)}, p=0.838\right)$ and age (mean \pm SD: $46.47 \pm 11.07$ vs $46.02 \pm 11.62$ years, $p$ $=0.849$ ). Table 6 shows comparisons concerning demographic and clinical variables. Lormetazepam was more likely used by mono- than poly-therapy users, vice versa zolpidem. BZD/Z-drug drops were more likely used by mono- than poly-therapy users. Mono-therapy users showed statistically significant lower daily dose (mean \pm SD: $104.63 \pm 200.46$ vs $105.42 \pm 263.86 \mathrm{mg}, p<0.001$ ), higher DDD (mean \pm SD: $56.32 \pm 66.79$ vs $33.26 \pm 39.61, p<0.001$ ), older age of first use (mean \pm SD: $31.67 \pm 10.26$ vs 28.75 \pm 11.59 years, $p=0.027$ ), lower duration of use (mean \pm SD: $88.34 \pm 85.76$ vs $139.38 \pm 124.93$ months, $p=0.002)$ than poly-therapy users. The two groups did not differ for diazepam equivalent dose (354.65 \pm 585.06 vs $305.13 \pm 266.90, p=0.487$ ).

Table 7 shows the hierarchical multiple regression analyses testing the risk of being mono-therapy users versus poly-therapy users on the whole sample and on groups of patients with current SUDs, lifetime SUDs, current and lifetime SUDs, or without SUDs.

In the whole sample, the predicting variables explained $14.1 \%$ of the overall variance $\left(\chi_{(\mathrm{df})}^{2}=57.65_{(11)}\right.$, $p<0.001$ ). Step 1 variables (i.e., sex, working activity, education, lifetime alcohol use, current or lifetime barbiturates use) explained $3.3 \%$ of the overall variance $\left(R^{2}=0.033, \chi_{(\mathrm{df})}^{2}=13.26_{(5)} p=0.021\right)$. Step 2 variable (duration of drug use, in months) predicted an additional $1.8 \%$ of unique variance $\left(R^{2}=0.051\right.$, $\left.\chi_{(\mathrm{df})}^{2}=7.18_{(1)}, p=0.007\right)$. Long-term drug use was less likely among mono- than poly-therapy users. Step 3 variable (diazepam equivalent dose) $\left(\chi_{(\mathrm{df})}^{2}=2.47_{(1)}, p=0.116\right)$ and Step 4 variable (daily dose) 
Table 5

Comparison between mono-therapy users $(n=155)$ and poly-therapy users $(n=28)$ among subjects with current and lifetime substance use disorder. Chi-square and Fisher test

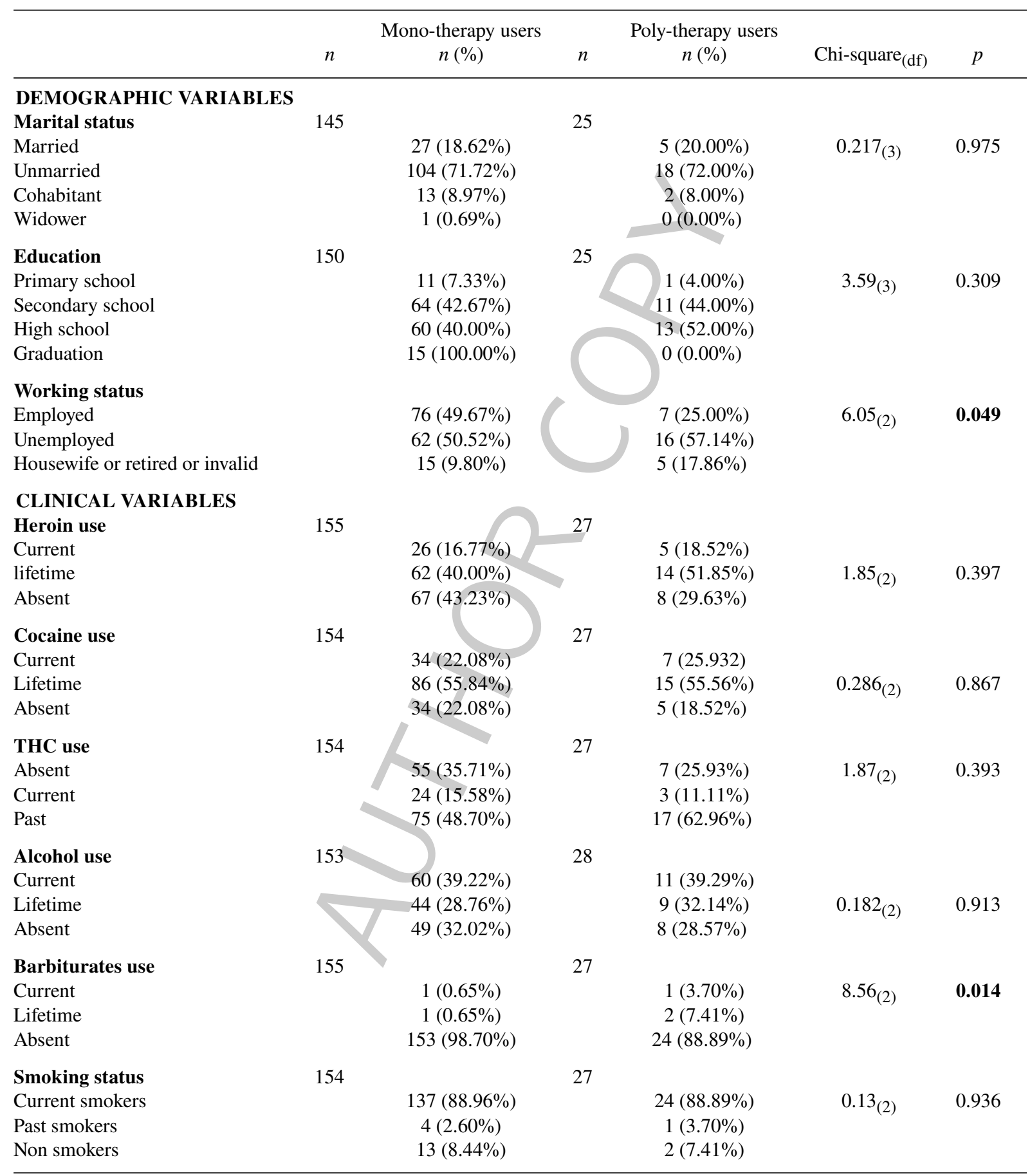


Table 5 (Continued).

\begin{tabular}{|c|c|c|c|c|c|c|}
\hline & $n$ & $\begin{array}{l}\text { Mono-therapy users } \\
n(\%)\end{array}$ & $n$ & $\begin{array}{c}\text { Poly-therapy users } \\
n(\%)\end{array}$ & Chi-square $_{(\mathrm{df})}$ & $p$ \\
\hline $\begin{array}{l}\text { Current psychiatry disorder } \\
\text { Present } \\
\text { Absent }\end{array}$ & 155 & $\begin{array}{c}141(90.97 \%) \\
14(9.03 \%)\end{array}$ & 28 & $\begin{array}{c}27(96.43 \%) \\
1(3.57 \%)\end{array}$ & $0.940_{(1)}$ & $0.474^{\mathrm{a}}$ \\
\hline $\begin{array}{l}\text { BZD used } \\
\text { Lormetazepam } \\
\text { Yes } \\
\text { No }\end{array}$ & 155 & $\begin{array}{l}87(56.13 \%) \\
68(43.87 \%)\end{array}$ & 28 & $\begin{array}{l}15(53.57 \%) \\
13(46.43 \%)\end{array}$ & $0.63_{(1)}$ & 0.838 \\
\hline $\begin{array}{l}\text { Lorazepam } \\
\text { Yes } \\
\text { No }\end{array}$ & 155 & $\begin{array}{c}21(13.55 \%) \\
134(86.45 \%)\end{array}$ & 28 & $\begin{array}{l}13(46.43 \%) \\
15(53.57 \%)\end{array}$ & $16.95_{(1)}$ & $<0.001$ \\
\hline $\begin{array}{l}\text { Alprazolam } \\
\text { Yes } \\
\text { No }\end{array}$ & 155 & $\begin{array}{c}10(6.45 \%) \\
145(93.55 \%)\end{array}$ & & $\begin{array}{r}6(21.43 \%) \\
22(78.57 \%)\end{array}$ & $6.67_{(1)}$ & $0.020^{\mathrm{a}}$ \\
\hline $\begin{array}{l}\text { Clonazepam } \\
\text { Yes } \\
\text { No }\end{array}$ & 155 & $\begin{array}{c}6(3.87 \%) \\
149(96.13 \%)\end{array}$ & 28 & $\begin{array}{c}5(17.86 \%) \\
23(82.14 \%)\end{array}$ & $8.21_{(1)}$ & $0.014^{\mathrm{a}}$ \\
\hline $\begin{array}{l}\text { Bromazepam } \\
\text { Yes } \\
\text { No }\end{array}$ & 155 & $\begin{array}{c}7(4.52 \%) \\
148(95.48 \%)\end{array}$ & 28 & $\begin{array}{c}4(14.29 \%) \\
24(85.71 \%)\end{array}$ & $4.01_{(1)}$ & 0.068 \\
\hline $\begin{array}{l}\text { Triazolam } \\
\text { Yes } \\
\text { No }\end{array}$ & 155 & $\begin{array}{c}5(3.23 \%) \\
150(96.77 \%)\end{array}$ & 28 & $\begin{array}{c}4(14.29 \%) \\
24(85.71 \%)\end{array}$ & $6.20_{(1)}$ & $0.032^{\mathrm{a}}$ \\
\hline $\begin{array}{l}\text { Diazepam } \\
\text { Yes } \\
\text { No }\end{array}$ & 155 & $\begin{array}{c}2(1.29 \%) \\
153(98.71 \%)\end{array}$ & 28 & $\begin{array}{c}3(10.71 \%) \\
25(89.29 \%)\end{array}$ & $7.92_{(1)}$ & $\mathrm{O.026}_{(1)}$ \\
\hline $\begin{array}{l}\text { Delorazepam } \\
\text { Yes } \\
\text { No }\end{array}$ & 155 & $\begin{array}{c}1(0.65 \%) \\
154(99.35 \%)\end{array}$ & 28 & $\begin{array}{c}3(10.71 \%) \\
25(89.29 \%)\end{array}$ & $11.25_{(1)}$ & $0.012^{\mathrm{a}}$ \\
\hline $\begin{array}{l}\text { Flurazepam } \\
\text { Yes } \\
\text { No }\end{array}$ & 155 & $\begin{array}{c}0(0.00 \%) \\
155(100.00 \%)\end{array}$ & 28 & $\begin{array}{c}3(10.71 \%) \\
25(89.29 \%)\end{array}$ & $16.88_{(1)}$ & $\mathbf{0 . 0 0 3}^{\mathrm{a}}$ \\
\hline $\begin{array}{l}\text { Etizolam } \\
\text { Yes } \\
\text { No }\end{array}$ & 155 & $\begin{array}{c}1(0.65 \%) \\
154(99.35 \%)\end{array}$ & 28 & $\begin{array}{c}0(0.00 \%) \\
28(100.00 \%)\end{array}$ & $0.18_{(1)}$ & $1.000^{\mathrm{a}}$ \\
\hline $\begin{array}{l}\text { Flunitrazepam } \\
\text { Yes } \\
\text { No }\end{array}$ & 155 & $\begin{array}{c}0(0.00 \%) \\
155(100.00 \%)\end{array}$ & 28 & $\begin{array}{c}0(0.00 \%) \\
28(100.00 \%)\end{array}$ & - & - \\
\hline
\end{tabular}


Table 5 (Continued).

\begin{tabular}{|c|c|c|c|c|c|c|}
\hline & $n$ & $\begin{array}{l}\text { Mono-therapy users } \\
n(\%)\end{array}$ & $n$ & $\begin{array}{c}\text { Poly-therapy users } \\
n(\%)\end{array}$ & Chi-square $_{(\mathrm{df})}$ & $p$ \\
\hline Oxazepam & 155 & & 28 & & & \\
\hline Yes & & $0(0.00 \%)$ & & $0(0.00 \%)$ & - & - \\
\hline No & & $155(100.00 \%)$ & & $28(100.00 \%)$ & & \\
\hline Temazepam & 155 & & 28 & & & \\
\hline Yes & & $0(0.00 \%)$ & & $0(0.00 \%)$ & - & - \\
\hline No & & $155(100.00 \%)$ & & $28(100.00 \%)$ & & \\
\hline Brotizolam & 155 & & 28 & & & \\
\hline Yes & & $1(0.65 \%)$ & & $0(0.00 \%)$ & $0.18_{(1)}$ & $1.00^{\mathrm{a}}$ \\
\hline No & & $154(99.35 \%)$ & & $28(100.00 \%)$ & & \\
\hline Prazepam & 155 & & 8 & & & \\
\hline Yes & & $1(0.65 \%)$ & & $0(0.00 \%)$ & $0.18_{(1)}$ & $1.000^{\mathrm{a}}$ \\
\hline No & & $154(99.35 \%)$ & & $28(100.00 \%)$ & & \\
\hline \multicolumn{7}{|l|}{ Z-drugs used } \\
\hline Zolpidem & 155 & & 28 & & & \\
\hline Yes & & $12(7.74 \%)$ & & $6(21.43 \%)$ & $5.01_{(1)}$ & $0.037^{\mathrm{a}}$ \\
\hline No & & $143(92.26 \%)$ & & $22(78.57 \%)$ & & \\
\hline Zoplicone & 155 & & 28 & & & \\
\hline Yes & & $1(0.65 \%)$ & & $1(3.57 \%)$ & $1.87_{(1)}$ & $0.283^{\mathrm{a}}$ \\
\hline No & & $154(99.35 \%)$ & & $27(96.43 \%)$ & & \\
\hline $\begin{array}{l}\text { Formulation of } \mathrm{BZD} / \mathrm{Z} \text {-drugs } \\
\text { used }\end{array}$ & 155 & & 28 & & & \\
\hline Tablets & & $46(29.68 \%)$ & & $15(53.57 \%)$ & $6.09_{(1)}$ & 0.014 \\
\hline Drops & & $109(70.32 \%)$ & & $13(46.43 \%)$ & & \\
\hline
\end{tabular}

Note: BZD: benzodiazepine; Z-drugs: non-benzodiazepine receptor agonists; a = Fisher test.

$\left(\chi_{(\mathrm{df})}^{2}=0.77_{(1)}, p=0.380\right)$ did not significantly increase the predictive ability of the model beyond Steps 2 and 3. Step 5 variable (DDD) predicted an additional $2.8 \%$ of unique variance $\left(R^{2}=0.083, \chi_{(\mathrm{df})}^{2}=9.59_{(1)}\right.$, $p=0.002)$. Higher DDD was more likely among mono-therapy than poly-therapy users. Step 6 variable (age of first use) predicted an additional $1.5 \%$ of unique variance $\left(R^{2}=0.098, \chi_{(\mathrm{df})}^{2}=6.30_{(1)}, p=0.012\right)$. First use of BDZs/Z-drugs at later age was more likely among mono- than poly-therapy users. Step 7 variable (formulation) predicted an additional $4.3 \%$ of unique variance $\left(R^{2}=0.141, \chi_{(\mathrm{df})}^{2}=18.06_{(1)}, p<\right.$ $0.001)$. BZD/Z-drug drops were more likely used by mono- than poly-therapy users.

Among subjects with current SUDs, the variables analysed did not significantly influence the risk of being mono-therapy or poly-therapy users $\left(\chi_{(\mathrm{df})}^{2}=11.33_{(10)}, p=0.332\right)$ (Table 7).

Among subjects with lifetime SUDs, the predicting variables explained $27.5 \%$ of the overall variance $\left(\chi_{(\mathrm{df})}^{2}=32.73_{(10)}, p<0.001\right)$. Step 1 variables (i.e., sex, working activity, education, lifetime alcohol use) explained $10.3 \%$ of the overall variance $\left(R^{2}=0.103, \chi_{(\mathrm{df})}^{2}=11.63_{(4)}, p=0.020\right)$. Step 2 variable (duration of drug use) $\left(\chi_{(\mathrm{df})}^{2}=0.49_{(1)}, p=0.484\right)$, Step 3 variable (diazepam equivalent dose $)\left(\chi_{(\mathrm{df})}^{2}=0.95_{(1)}, p=\right.$ $0.329)$, Step 4 variable (daily dose) $\left(\chi_{(\mathrm{df})}^{2}=0.50_{(1)}, p=0.445\right)$, Step 5 variable (DDD) $\left(\chi_{(\mathrm{df})}^{2}=1.91_{(1)}, p=\right.$ 0.167 ) did not significantly increase the predictive ability of the model previous steps. Step 6 variable (age 
Table 6

Comparison between mono-therapy users $(n=404)$ and poly-therapy users $(n=56)$ among subjects without substance use disorder. Chi-square and Fisher test

\begin{tabular}{|c|c|c|c|c|c|c|}
\hline & $n$ & $\begin{array}{c}\text { Mono-therapy users } \\
n(\%)\end{array}$ & $n$ & $\begin{array}{c}\text { Poly-therapy users } \\
n(\%)\end{array}$ & Chi-square $_{(\mathrm{df})}$ & $p$ \\
\hline \multicolumn{7}{|c|}{ DEMOGRAPHIC VARIABLES } \\
\hline Marital status & 362 & & 46 & & & \\
\hline Married & & $151(41.72 \%)$ & & $16(34.78 \% \%)$ & $1.79_{(3)}$ & 0.616 \\
\hline Unmarried & & $172(47.51 \% \%)$ & & $23(50.00 \%)$ & & \\
\hline Cohabitant & & $32(8.34 \% \%)$ & & $5(10.87 \%)$ & & \\
\hline Widower & & $7(1.93 \%)$ & & $2(4.35 \%)$ & & \\
\hline Education & 376 & & 50 & & & \\
\hline Primary school & & $21(5.58 \%)$ & & $5(10.00 \%)$ & $6.64_{(1)}$ & 0.084 \\
\hline Secondary school & & $96(25.53 \%)$ & & $16(32.00 \%)$ & & \\
\hline High school & & $167(44.41 \%)$ & & $13(26.00 \%)$ & & \\
\hline Graduation & & $92(24.47 \%)$ & & $16(32.00 \%)$ & & \\
\hline Working status & 388 & & & & & \\
\hline Employed & & $212(54.64 \%)$ & & $24(45.28 \%)$ & $1.88_{(2)}$ & 0.390 \\
\hline Unemployed & & $87(22.42 \%)$ & & $13(24.53 \%)$ & & \\
\hline Housewife or retired or invalid & & $89(22.94 \%)$ & & $16(30.19 \%)$ & & \\
\hline \multicolumn{7}{|l|}{ CLINICAL VARIABLES } \\
\hline Smoking status & 391 & & 54 & & & \\
\hline Current smokers & & $182(46.55 \%)$ & & $26(48.15 \%)$ & $2.55_{(2)}$ & 0.279 \\
\hline Past smokers & & $34(8.69 \%)$ & & $8(14.81 \%)$ & & \\
\hline Non smokers & & $175(44.76 \%)$ & & $20(37.04 \%)$ & & \\
\hline Current psychiatry disorder & 404 & & 56 & & & \\
\hline Present & & $368(91.09 \%)$ & & $53(94.64 \%)$ & $0.80_{(1)}$ & 0.371 \\
\hline Absent & & $36(8.91 \%)$ & & $3(5.36 \%)$ & & \\
\hline \multicolumn{7}{|l|}{ BZD used } \\
\hline Lormetazepam & 404 & & 56 & & & \\
\hline Yes & & $263(65.10 \%)$ & & $24(42.86 \%)$ & $10.37(1)$ & 0.001 \\
\hline No & & $141(34.90 \%)$ & & $32(57.14 \%)$ & & \\
\hline Lorazepam & 404 & & 56 & & & \\
\hline Yes & & $38(9.41 \%)$ & & $18(32.14 \%)$ & $23.78_{(1)}$ & $<0.001$ \\
\hline No & & $366(90.59 \%)$ & & $38(67.86 \%)$ & & \\
\hline Alprazolam & 40 & & 56 & & & \\
\hline Yes & & $33(8.17 \%)$ & & $18(32.14 \%)$ & $28.68_{(1)}$ & $<0.001$ \\
\hline No & & $371(91.83 \%)$ & & $38(67.86 \%)$ & & \\
\hline Clonazepam & 404 & & 56 & & & \\
\hline Yes & & $3(0.74 \%)$ & & $9(16.07 \%)$ & $45.49_{(1)}$ & $<0.001^{\mathrm{a}}$ \\
\hline No & & $401(99.26 \%)$ & & $47(83.93 \%)$ & & \\
\hline Bromazepam & 404 & & 56 & & & \\
\hline Yes & & $13(3.22 \%)$ & & $7(12.50 \%)$ & $10.18_{(1)}$ & 0.001 \\
\hline No & & $391(96.78 \%)$ & & $49(87.50 \%)$ & & \\
\hline
\end{tabular}


Table 6 (Continued).

\begin{tabular}{|c|c|c|c|c|c|c|}
\hline & $n$ & $\begin{array}{c}\text { Mono-therapy users } \\
n(\%)\end{array}$ & $n$ & $\begin{array}{c}\text { Poly-therapy users } \\
n(\%)\end{array}$ & Chi-square $_{(\mathrm{df})}$ & $p$ \\
\hline Triazolam & 404 & & 56 & & & \\
\hline Yes & & $3(0.74 \%)$ & & $10(17.86 \%)$ & $52.46_{(1)}$ & $<0.001^{\mathrm{a}}$ \\
\hline No & & $401(99.26 \%)$ & & $46(82.14 \%)$ & & \\
\hline Diazepam & 404 & & 56 & & & \\
\hline Yes & & $6(1.49 \%)$ & & $9(16.07 \%)$ & $33.17_{(1)}$ & $<0.001$ \\
\hline No & & $398(98.51 \%)$ & & $47(83.93 \%)$ & & \\
\hline Delorazepam & 404 & & 56 & & & \\
\hline Yes & & $3(0.74 \%)$ & & $6(10.71 \%)$ & $25.49_{(1)}$ & $<0.001^{\mathrm{a}}$ \\
\hline No & & $401(99.26 \%)$ & & $50(89.29 \%)$ & & \\
\hline Flurazepam & 404 & & 56 & & & \\
\hline yes & & $0(0.00 \%)$ & & $5(8.93 \%)$ & $36.47^{(1)}$ & $<0.001^{\mathrm{a}}$ \\
\hline No & & $404(100.00 \%)$ & & $51(91.07 \%)$ & & \\
\hline Etizolam & 404 & & & & & \\
\hline Yes & & $3(0.74 \%)$ & & $0(0.00 \%)$ & $0.42_{(1)}$ & $1.000^{\mathrm{a}}$ \\
\hline No & & $401(99.26 \%)$ & & $56(100.00 \%)$ & & \\
\hline Flunitrazepam & 404 & & 56 & & & \\
\hline Yes & & $0(0.00 \%)$ & & $2(3.57 \%)$ & $14.49_{(1)}$ & $0.015^{\mathrm{a}}$ \\
\hline No & & $404(100.00 \%)$ & - & $54(96.43 \%)$ & & \\
\hline Oxazepam & 404 & & 56 & & & \\
\hline Yes & & $0(0.00 \%)$ & & $1(1.79 \%)$ & $7.23_{(1)}$ & $0.122^{\mathrm{a}}$ \\
\hline No & & $404(100.00 \%)$ & & $55(98.21 \%)$ & & \\
\hline Temazepam & 404 & & 56 & & & \\
\hline Yes & & $1(0.25 \%)$ & & $1(1.79 \%)$ & $2.68_{(1)}$ & $0.229^{\mathrm{a}}$ \\
\hline No & & 403 (99.75\%) & & $55(98.21 \%)$ & & \\
\hline Brotizolam & 404 & & 56 & & & \\
\hline Yes & & $0(0.00 \%)$ & & $0(0.00 \%)$ & - & - \\
\hline No & & $404(100.00 \%)$ & & $56(100.00 \%)$ & & \\
\hline Prazepam & 404 & & 56 & & & \\
\hline Yes & & $0(0.00 \%)$ & & $0(0.00 \%)$ & - & - \\
\hline No & & $404(100.00 \%)$ & & $56(100.00 \%)$ & & \\
\hline \multicolumn{7}{|l|}{ Z-drugs used } \\
\hline Zolpidem & 404 & & 56 & & & \\
\hline Yes & & $36(8.91 \%)$ & & $13(23.21 \%)$ & $10.57_{(1)}$ & 0.001 \\
\hline No & & $368(91.09 \%)$ & & $43(76.79 \%)$ & & \\
\hline Zoplicone & 404 & & 56 & & & \\
\hline Yes & & $1(0.25 \%)$ & & $0(0.00 \%)$ & $0.14_{(2)}$ & $1.000^{\mathrm{a}}$ \\
\hline No & & $403(99.75 \%)$ & & $56(100.00 \%)$ & & \\
\hline
\end{tabular}


Table 6 (Continued).

\begin{tabular}{|c|c|c|c|c|c|c|}
\hline & $n$ & $\begin{array}{c}\text { Mono-therapy users } \\
n(\%)\end{array}$ & $n$ & $\begin{array}{l}\text { Poly-therapy users } \\
n(\%)\end{array}$ & Chi-square $_{(\mathrm{df})}$ & $p$ \\
\hline $\begin{array}{l}\text { Formulation of BZD/Z-drugs } \\
\text { used }\end{array}$ & 404 & & 56 & & & \\
\hline Tablets & & $93(23.02 \%)$ & & $29(51.79 \%)$ & $20.88_{(1)}$ & $<0.001$ \\
\hline Drops & & $311(77.98 \%)$ & & $27(48.21 \%)$ & & \\
\hline
\end{tabular}

Note: BZD: benzodiazepine; Z-drugs: non-benzodiazepine receptor agonists; a = Fisher test.

of first use) predicted an additional $4.2 \%$ of unique variance $\left(R^{2}=0.178, \chi_{(\mathrm{df})}^{2}=5.05_{(1)}, p=0.025\right)$. First use of BDZs/Z-drugs at later age was more likely among mono- than poly-therapy users. Step 7 variable (formulation) predicted an additional 9.7\% of unique variance $\left(R^{2}=0.275, \chi_{(\mathrm{df})}^{2}=12.26_{(1)}, p<0.001\right)$. BZD/Z-drug drops were more likely used by mono- than poly-therapy users (Table 7).

Among subjects with current and lifetime SUDs, the variables analysed did not significantly influence the risk of being mono- or poly-therapy users $\left(\chi_{(\mathrm{df})}^{2}=11.29_{(11)}, p=0.419\right)$ (Table 7).

Among subjects without SUDs, the predicting variables explained $16.2 \%$ of the overall variance $\left(\chi_{(\mathrm{df})}^{2}=29.37_{(9)}, p=0.001\right)$. Step 1 variables (i.e., sex, working activity, education) did not significantly increase the predictive ability of the model $\left(\chi_{(\mathrm{df})}^{2}=4.41_{(3)}, p=0.22\right)$. Step 2 variable (duration of drug use) predicted an additional $5.5 \%$ of unique variance $\left(R^{2}=0.080, \chi_{(\mathrm{df})}^{2}=9.73_{(1)}, p=0.002\right)$. Long-term drug use was less likely among mono- than poly-therapy users. Step 3 variable (diazepam equivalent dose) $\left(\chi_{(\mathrm{df})}^{2}=0.01_{(1)}, p=0.927\right)$ and Step 4 variable (daily dose $)\left(\chi_{(\mathrm{df})}^{2}=0.25_{(1)}, p=0.618\right)$ did not significantly increase the predictive ability of the model beyond Steps 2 and 3, respectively. Step 5 variable (DDD) predicted an additional $5.7 \%$ of unique variance $\left(R^{2}=0.138, \chi_{(\mathrm{df})}^{2}=10.39_{(1)}, p=0.001\right)$. Higher DDD was more likely among mono- than poly-therapy users. Step 6 variable (age of first use) $\left(\chi_{(\mathrm{df})}^{2}=0.94_{(1)}\right.$, $p=0.332)$ and Step 7 variable (formulation) $\left(\chi_{(\mathrm{df})}^{2}=3.64_{(1)}, p=0.057\right)$ did not significantly increase the predictive ability of the model beyond previous steps (Table 7).

\section{Discussion}

The present study suggests that mono- and poly-therapy users have different patterns of BZD/Z-drug use. In the whole sample, mono-therapy users used less frequently zolpidem [4] (which was observed among current SUDs subjects, lifetime SUDs subjects, current and lifetime SUDs subjects, subjects without SUDs, thus it seems strictly related to the poly-therapy condition), had lower daily dose and older age of first BDZ/Z-drug use [4,6,30]. In addition, lormetazepam was more frequently used in monotherapy, this result was maintained among subjects without SUDs and might be explained by a relatively low dependence liability and low risk to produce hang-over effects of lormetazepam [31-34]. Drops were more likely used by mono-therapy users, which is explained by the high prevalence of lormetazepam use in drops [35]. Similar findings were observed among current SUDs subjects, lifetime SUDs subjects, current and lifetime SUDs subjects, and subjects without SUDs. Mono-therapy users had higher DDD, which might be related to the fact that patients may try to manage the symptoms of their original disease as well as withdrawal manifestations increasing the dose of the drug that they are using rather than adding other drugs. Similar results were found among lifetime SUDs subjects as well as non-SUDs subjects. Among current SUDs subjects, mono-therapy users had lower diazepam equivalent dose and older age of 
G. Mansueto et al. / Mono- and poly-therapy with benzodiazepines or Z-drugs

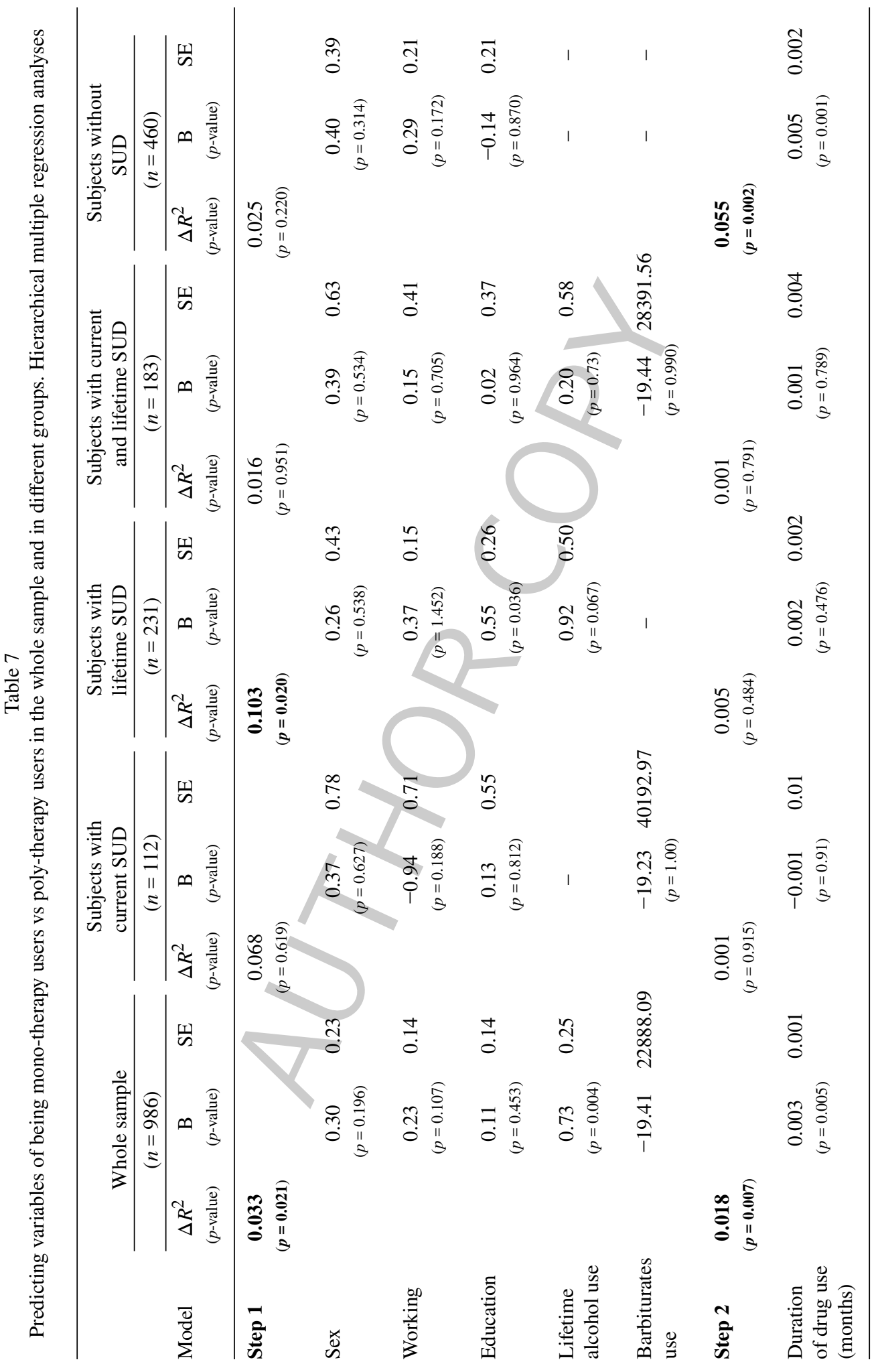




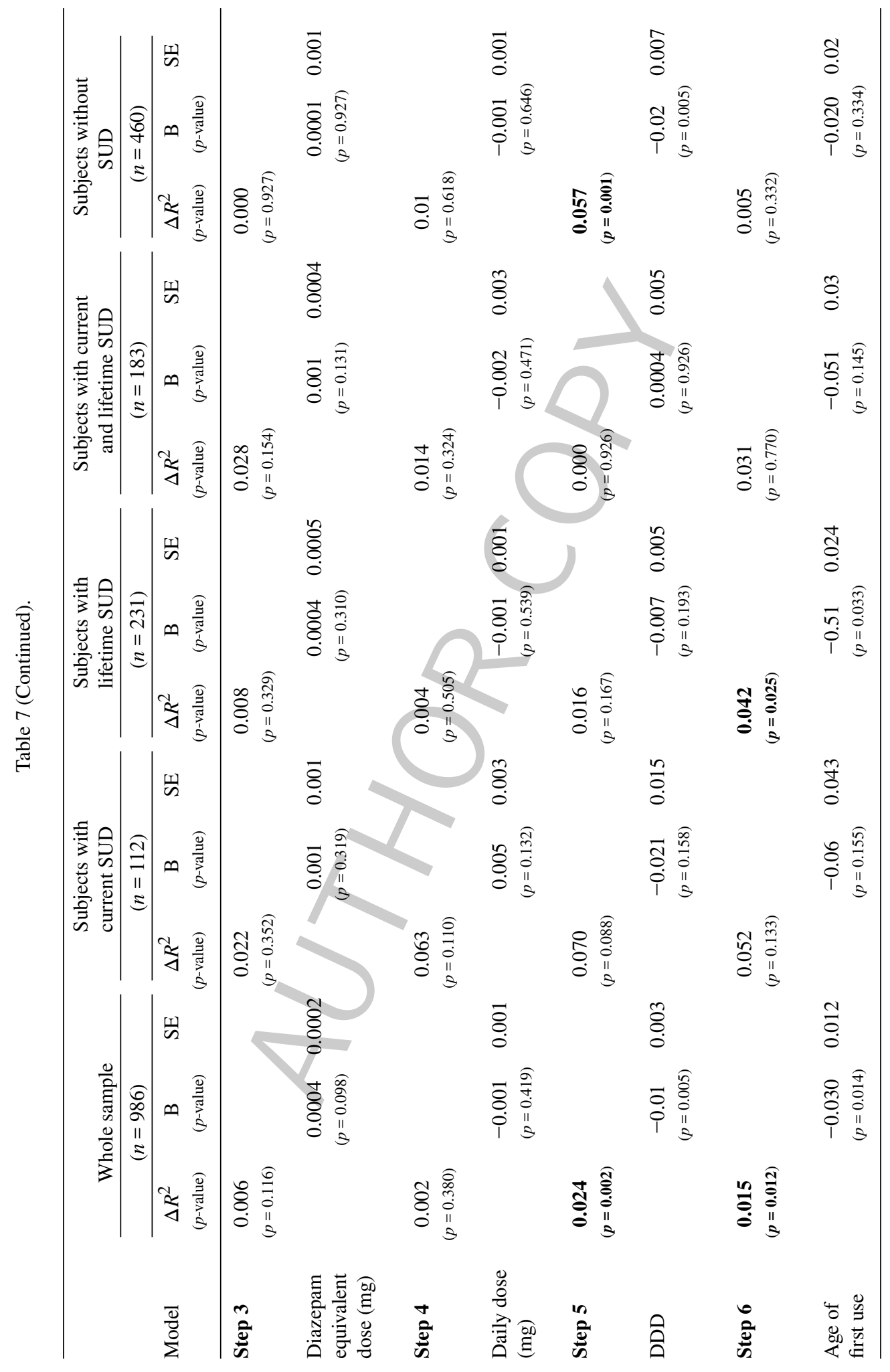




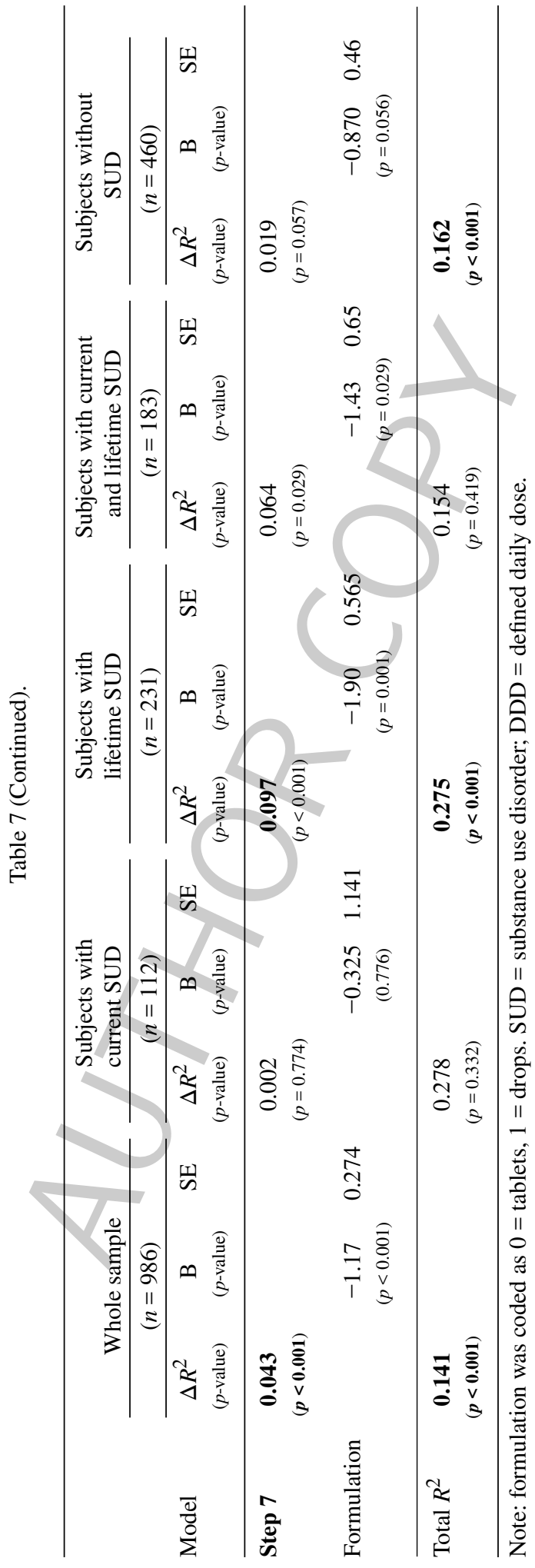


first use $[4,6,30]$. Among subjects without SUDs, mono-therapy users had lower daily dose, older age of first use, and lower duration of use [4,6,30].

The variance of being mono- vs poly-therapy users was explained by several variables: BZD/Z-drugs formulation, followed by DDD, duration of drug use, age of first use. The results on drug formulation are new in the literature and suggest that tablets should be used with caution since subjects assuming zolpidem by tablets had around 2-fold increase risk of problem use than those using liquid formulation [36].

The result on DDD seems consistent to the rationale that using lower doses of two or more drugs could achieve efficacy with less severe side effects than would be expected from higher dosage of a single drug [37-39]. However, there is no evidence that poly-therapy had lower side effects than monotherapy [10-12], thus limiting BZD/Z-drugs dose is crucial [25]. Findings on duration of BZD/Z-drug use and on age of first use support current recommendations to limit their prescription, the duration of use, and to delay the beginning of these treatments as much as possible.

Among subjects with current SUDs as well as among subjects with current and lifetime SUDs, the variables analysed did not explain the variance of being mono-or poly-therapy users. Among subjects with lifetime SUDs, the variance of being mono- vs poly-therapy users was explained by BZD/Z-drugs formulation followed by age of first use. Thus, the use of tablets and a prescription at early age should be carefully evaluated in this population. Finally, among non-SUDs subjects, the variance of being monoversus poly-therapy users was explained by DDD and duration of use. Once again, limiting the dose [25] and the duration of treatment [40] seem crucial.

The present research has some limitations. First, subjects were enrolled at a tertiary care clinic limiting generalizability of results. Second, some variables were assessed retrospectively, thus a recall bias could not be excluded. Third, this is an observational study and was not possible to establish a causal relationship between the variables under study and the status of mono-/poly-therapy user. Finally, variables other than those here considered (e.g., medical illness, compliance, illness behaviour, personality) $[11,13]$ may have influenced the risk of being mono- vs poly-therapy users. For these reasons, future research involving several single tertiary care clinics, applying a longitudinal design, and assessing other potential confounding variables are warranted.

\section{Conclusions}

Lormetazepam appears less likely associated to poly-therapy than other benzodiazepines while zolpidem seems more likely associated. Tablets, high drug doses, long duration of treatment, and early age of first use were more likely associated to poly- than mono-therapy. Interestingly, such variables differently explained the variance of being mono- versus poly-therapy users when the hierarchical multivariate regression analyses were run stratifying for groups of subjects. In particular, among subjects with current SUDs and among subjects with current and lifetime SUDs, the variables analysed did not explain the variance of being mono-or poly-therapy users. Among subjects with lifetime SUDs, the variance was explained by BZD/Z-drugs formulation followed by age of first use while, among non-SUDs subjects, the variance was explained by DDD and duration of use. This suggests that patients have different clinical features and a pharmacological prescription should be tailored to them also based on the variables here analysed. Once again, it is important not to refer to the "average patients" [41], for whom differences in terms of severity of symptoms, comorbidity and other clinical nuances are neglected, but to consider any variable which may demarcate prognostic and therapeutic differences [41] among patients who otherwise may be deceptively considered similar only because they share the same diagnostic label or clinical problem. 


\section{Conflict of interest}

The authors report no conflict of interest.

\section{References}

[1] Cloos JM, Bocquet V, Rolland-Portal I, Koch P, Chouinard G. Hypnotics and triazolobenzodiazepines-best predictors of high-dose benzodiazepine use: Results from the Luxembourg National Health Insurance Registry. Psychother Psychosom. 2015;84(5):273-83. doi:10.1159/000434755.

[2] Brandt J, Alessi-Severini S, Singer A, Leong C. Novel measures of benzodiazepine and Z-Drug utilisation trends in a Canadian provincial adult population (2001-2016). J Popul Ther Clin Pharmacol. 2019;26(1):e22-38. doi:10.22374/17106222.26.1.3.

[3] Zandstra SM, Furer JW, van de Lisdonk EH, van't HM, Bor JH, van Weel C et al. Different study criteria affect the prevalence of benzodiazepine use. Soc Psychiatry Psychiatr Epidemiol. 2002;37(3):139-44. doi:10.1007/s001270200006.

[4] Sakshaug S, Handal M, Hjellvik V, Berg C, Ripel Å, Gustavsen I et al. Long-term use of Z hypnotics and co-medication with benzodiazepines and opioids. Basic Clin Pharmacol Toxicol. 2017;120(3):292-8. doi:10.1111/bcpt.12684.

[5] Schifano F, Chiappini S, Corkery JM, Guirguis A. An insight into Z-drug abuse and dependence: An examination of reports to the European Medicines Agency Database of Suspected Adverse Drug Reactions. Int J Neuropsychol. 2019;22(4):270-7. doi:10.1093/ijnp/pyz007.

[6] Petitjean S, Ladewig D, Meier CR, Amrein R, Wiesbeck GA. Benzodiazepine prescribing to the Swiss adult population: Results from a national survey of community pharmacies. Int Clin Psychopharmacol. 2007;22(5):292-8 . doi:10.1097/yic.0b013e328105e0f2.

[7] Cadogan CA, Ryan C, Cahir C, Bradley CP, Bennett K. Benzodiazepine and Z-drug prescribing in Ireland: Analysis of national prescribing trends from 2005 to 2015. Br J Clin Pharmacol. 2018;84(6):1354-63. doi:10.1111/bcp.13570.

[8] Kurko TAT, Saastamoinen LK, Tähkäpää S, Tuulio-Henriksson A, Taiminen T, Tiihonen J et al. Long-term use of benzodiazepines: Definitions, prevalence and usage patterns-a systematic review of register-based studies. Eur Psychiatry. 2015;30(8):1037-47. doi:10.1016/j.eurpsy.2015.09.003.

[9] Tjagvad C, Clausen T, Handal M, Skurtveit S. Benzodiazepine prescription for patients in treatment for drug use disorders: A nationwide cohort study in Denmark, 2000-2010. BMC Psychiatry. 2016;16(168):1-12. doi:10.1186/s12888016-0881-y.

[10] Lindsey WT, Stewart D, Childress D. Drug interactions between common illicit drugs and prescription therapies. Am J Drug Alcohol Abuse. 2012;38(4):334-43. doi:10,3109/00952990.2011.643997.

[11] Kukreja S, Kalra G, Shah N, Shrivastava A. Polypharmacy in psychiatry: A review. Mens Sana Monogr. 2013;11(1):82-99. doi:10.4103/0973-1229.104497.

[12] Sung HG, Li J, Nam JH, Won DY, Choi B, Shin JY. Concurrent use of benzodiazepines, antidepressants, and opioid analgesics with zolpidem and risk for suicide: A case-control and case-crossover study. Soc Psychiatry Psychiatr Epidemiol. 2019;54(12):1535-44. doi:10.1007/s00127-019-01713-x.

[13] Vozoris NT. Benzodiazepine and opioid co-usage in the US population, 1999-2014: An exploratory analysis. Sleep. 2019;42(4):zsy264. doi:10.1093/sleep/zsy264.

[14] American Psychiatric Association. Diagnostic and Statistical Manual of Mental Disorders 4th ed. (DSM-IV-TR). Washington DC: American Psychiatric Association; 2000.

[15] Janhsen K, Roser P, Hoffmann K. The problems of long-term treatment with benzodiazepines and related substances. Dtsch Arztebl Int. 2015;112(1-2):1-7. doi:10.3238/arztebl.2015.0001.

[16] Lugoboni F, Mirijello A, Morbioli L, Faccini M, Casari R, De Cosmo S et al. Zolpidem high-dose abuse: What about the liver? Results from a series of 107 patients. Expert Opin Drug Saf. 2019;18(8):753-8. doi:10.1080/14740338. 2019.1628216.

[17] Faccini M, Leone R, Opri S, Casari R, Resentera C, Morbioli L et al. Slow subcutaneous infusion of flumazenil for the treatment of long-term, high-dose benzodiazepine users: A review of 214 cases. J Psychopharmacol. 2016;30(10):1047-53. doi:10.1177/0269881116647505.

[18] First MB, Spitzer RL, Gibbon M, Williams JBW. Structured clinical interview for DSM-IV-TR Axis I disorders, patient edition (SCID-I/P). New York: Biometrics Research, New York State Psychiatric Institute; 2002.

[19] Mazzi F, Morosini P, De Girolamo G, Lussetti M, Guaraldi GP. SCID-I: Structured Clinical Interview for DSM-IV Axis I Disorders. Italian ed. Firenze: Giunti OS; 2000. 
[20] Zanarini M, Frankenburg F, Sickel A, Yong L. The Diagnostic Interview for DSM-IV Personality Disorders (DIPD-IV). Belmont: McLean Hospital; 1996.

[21] Ali Z, Bhaskar SB. Basic statistical tools in research and data analysis. Indian J Anaesth. 2016;60(9):662-9. doi:10.4103/ 0019-5049.190623.

[22] Barbaranelli C, D’Olimpio F. Analisi dei dati con SPSS, Vol. I. Milano: Led; 2007.

[23] Kim HY. Statistical notes for clinical researchers: Chi-squared test and Fisher's exact test. Restor Dent Endod. 2017;42(2):152-5. doi:10.5395/rde.2017.42.2.152.

[24] WHO Collaborating Centre for Drug Statistics Methodology (WHOCC). DDD definition and general considerations. Retrieved the 6th of November 2015. Available from: https://www.whocc.no/ddd/definition_and_general_considera/. Accessed 28 February, 2021.

[25] Brett J, Murnion B. Management of benzodiazepine misuse and dependence. Aust Prescr. 2015;38(5):152-5. doi:10.18773/ austprescr.2015.055.

[26] Inada T, Inagaki A. Psychotropic dose equivalence in Japan. Psychiatry Clin Neurosci. 2015;69(8):440-7. doi:10.1111/ pen.12275.

[27] World Health Organization (WHO). WHO Lexicon of Alcohol and Drug Terms. Geneva: WHO; 1994. Available from: https://www.who.int/substance_abuse/terminology/who_lexicon/en/. Accessed 28 February, 2021.

[28] Kozlowski LT, Wilkinson DA, Skinner W, Kent C, Franklin T, Pope M. Comparing tobacco cigarette dependence with other drug dependencies: Greater or equal 'difficulty quitting' and 'urges to use, 'but less' pleasure' from cigarettes. Jama. 1989;261(6):898-901. doi:10.1001/jama.261.6.898.

[29] Shmulewitz D, Greene ER, Hasin D. Commonalities and differences across substance use disorders: Phenomenological and epidemiological aspects. Alcohol Clin Exp Res. 2015;39(10):1878-900. doi:10.1111/acer.12838.

[30] Votaw VR, Geyer R, Rieselbach MM, McHugh RK. The epidemiology of benzodiazepine misuse: A systematic review. Drug Alcohol Depend. 2019;1(200):95-114. doi:10.1016/j.drugalcdep.2019.02.033.

[31] Faccini M, Leone R, Pajusco B, Quaglio G, Casari R, Albiero A et al. Lormetazepam addiction: Data analysis from an Italian medical unit for addiction. Risk Manag Healthc Policy. 2012;5:43-8. doi:10.2147/rmhp.s31745.

[32] Horowski R. Dependence liability of lormetazepam: Are all benzodiazepines equal? The case of the new i.v. lormetazepam for anesthetic procedures. J Neural Trans. 2020;127(8):1107-15. doi:10.1007/s00702-020-02209-8.

[33] Heidrich H, Ott H, Beach RC. Lormetazepam-a benzodiazepine derivative without hangover effect? A double-blind study with chronic insomniacs in a general practice setting. Int J Clin Pharmacol Ther Toxicol. 1981;19(1):11-7.

[34] Sastre Y, Hernández MS, Hentschel HD, Fichte K. Comparative efficacy of lormetazepam (Noctamid) and diazepam (Valium) in 100 out-patients with insomnia. J Int Med Res. 1981;9(3):199-202. doi:10.1177/030006058100900309.

[35] Faccini M, Tamburin S, Casari R, Morbioli L, Lugoboni F. High-dose lormetazepam dependence: Strange case of Dr. Jekyll and Mr. Hyde. Intern Emerg Med. 2019;14(8):1271-8. doi:10.1007/s11739-019-02101-8.

[36] Ferini-Strambi L, Sforza M, Galbiati A. High-dose lormetazepam dependence: Strange case of Dr. Jekyll and Mr. Hydecomment. Intern Emerg Med. 2020;15(2):341-2. doi:10.1007/s11739-019-02142-z.

[37] Hjorth P, Kilian R, Sørensen HØ, Eriksen SE, Davidsen AS, Jensen SO et al. Reducing psychotropic pharmacotherapy in patients with severe mental illness: A cluster-randomized controlled intervention study. Ther Adv Psychopharmacol. 2015;5(2):67-75. doi:10.1177/2045125314565361.

[38] Andreadis EA, Tsourous GI, Marakomichelakis GE, Katsanou PM, Fotia ME, Vassilopoulos CV et al. High-dose monotherapy vs low-dose combination therapy of calcium channel blockers and angiotensin receptor blockers in mild to moderate hypertension. J Hum Hypertens. 2005;19(6):491-6. doi:10.1038/sj.jhh.1001843.

[39] Neutel JM, Smith DH, Weber MA. Low dose combination therapy vs. high dose monotherapy in the management of hypertension. J Clin Hypertens (Greenwich). 1999;1(3):79-86.

[40] Vorspan F, Barré T, Pariente A, Montastruc F, Tournier M, Faut-il limiter la durée des traitements par benzodiazépines? Should the duration of treatment be limited using benzodiazepines? Presse Med, 47(10): 892-8, 2018. doi:10.1016/j.lpm.2018.10.006.

[41] Fava GA. Rational use of antidepressant drugs. Psychother Psychosom. 2014;83(4):197-204. doi:10.1159/000362803. 